\title{
The X-ray properties of $z>6$ quasars: no evident evolution of accretion physics in the first Gyr of the Universe
}

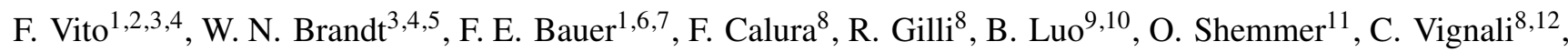 \\ G. Zamorani ${ }^{8}$, M. Brusa ${ }^{8,12}$, F. Civano ${ }^{13}$, A. Comastri ${ }^{8}$, and R. Nanni ${ }^{8,12}$ \\ 1 Instituto de Astrofisica and Centro de Astroingenieria, Facultad de Fisica, Pontificia Universidad Catolica de Chile, Casilla 306 , \\ Santiago 22, Chile \\ e-mail: fabio.vito@uc.cl \\ 2 Chinese Academy of Sciences South America Center for Astronomy, National Astronomical Observatories, CAS, \\ Beijing 100012, PR China \\ 3 Department of Astronomy \& Astrophysics, 525 Davey Lab, The Pennsylvania State University, University Park, PA 16802, USA \\ ${ }^{4}$ Institute for Gravitation and the Cosmos, The Pennsylvania State University, University Park, PA 16802, USA \\ 5 Department of Physics, The Pennsylvania State University, University Park, PA 16802, USA \\ 6 Millennium Institute of Astrophysics (MAS), Nuncio Monseñor Sótero Sanz 100, Providencia, Santiago, Chile \\ 7 Space Science Institute, 4750 Walnut Street, Suite 205, Boulder, CO 80301, USA \\ 8 INAF - Osservatorio di Astrofisica e Scienza dello Spazio di Bologna, Via Gobetti 93/3, 40129 Bologna, Italy \\ 9 School of Astronomy and Space Science, Nanjing University, Nanjing 210093, PR China \\ ${ }^{10}$ Key Laboratory of Modern Astronomy and Astrophysics (Nanjing University), Ministry of Education, Nanjing, Jiangsu 210093, \\ PR China \\ 11 Department of Physics, University of North Texas, Denton, TX 76203, USA \\ 12 Dipartimento di Fisica e Astronomia, Università degli Studi di Bologna, via Gobetti 93/2, 40129 Bologna, Italy \\ 1315 Center for Astrophysics | Harvard \& Smithsonian, 60 Garden St, Cambridge, MA 02138, USA
}

Received 1 July 2019 / Accepted 26 August 2019

\begin{abstract}
Context. X-ray emission from quasars (QSOs) has been used to assess supermassive black hole accretion properties up to $z \approx 6$. However, at $z>6$ only $\approx 15$ QSOs are covered by sensitive X-ray observations, preventing a statistically significant investigation of the X-ray properties of the QSO population in the first Gyr of the Universe.

Aims. We present new Chandra observations of a sample of $10 z>6$ QSOs, selected to have virial black-hole mass estimates from $\mathrm{Mg}$ II line spectroscopy $\left(\log \frac{M_{\mathrm{BH}}}{M_{\odot}}=8.5-9.6\right)$. Adding archival X-ray data for an additional $15 z>6$ QSOs, we investigate the X-ray properties of the QSO population in the first Gyr of the Universe. In particular, we focus on the $L_{\mathrm{UV}}-L_{\mathrm{X}}$ relation, which is traced by the $\alpha_{\text {ox }}$ parameter, and the shape of their X-ray spectra.

Methods. We performed photometric analyses to derive estimates of the X-ray luminosities of our $z>6$ QSOs, and thus their $\alpha_{\mathrm{ox}}$ values and bolometric corrections $\left(K_{\mathrm{bol}}=L_{\mathrm{bol}} / L_{\mathrm{X}}\right)$. We compared the resulting $\alpha_{\mathrm{ox}}$ and $K_{\mathrm{bol}}$ distributions with the results found for QSO samples at lower redshift, and ran several statistical tests to check for a possible evolution of the $L_{\mathrm{UV}}-L_{\mathrm{X}}$ relation. Finally, we performed a basic X-ray spectral analysis of the brightest $z>6$ QSOs to derive their individual photon indices, and joint spectral analysis of the whole sample to estimate the average photon index.

Results. We detect seven of the new Chandra targets in at least one standard energy band, while two more are detected discarding energies $E>5 \mathrm{keV}$, where background dominates. We confirm a lack of significant evolution of $\alpha_{\mathrm{ox}}$ with redshift, which extends the results from previous works up to $z>6$ with a statistically significant QSO sample. Furthermore, we confirm the trend of an increasing bolometric correction with increasing luminosity found for QSOs at lower redshifts. The average power-law photon index of our sample $\left(\langle\Gamma\rangle=2.20_{-0.34}^{+0.39}\right.$ and $\langle\Gamma\rangle=2.13_{-0.13}^{+0.13}$ for sources with $\langle 30$ and $>30$ net counts, respectively $)$ is slightly steeper than, but still consistent with, typical QSOs at $z=1-6$.

Conclusions. All of these results indicate a lack of substantial evolution of the inner accretion-disk and hot-corona structure in QSOs from low redshift to $z>6$. Our data hint at generally high Eddington ratios at $z>6$.
\end{abstract}

Key words. methods: data analysis - galaxies: active - galaxies: nuclei - X-rays: galaxies - galaxies: high-redshift quasars: general

\section{Introduction}

X-ray emission from accreting supermassive black holes (SMBHs), shining as quasars (QSOs), is thought to originate from inverse Compton scattering in the so-called "hot corona" of the UV/optical photons produced by the accretion disk via thermal emission (e.g., Galeev et al. 1979; Haardt \& Maraschi 1991; Beloborodov 2017). The relative importance of the hot corona and the accretion disk to the total radiative output is usually parametrized with $\alpha_{\text {ox }}=0.38 \times \log \left(L_{2 \mathrm{keV}} / L_{2500 \AA}\right)$, which represents the slope of a nominal power-law connecting the rest-frame UV and X-ray emission (e.g., Brandt \& Alexander 2015, and references therein). It is important to note that $\alpha_{\text {ox }}$ is known to anti-correlate with the QSO UV luminosity (e.g., Steffen et al. 2006; Just et al. 2007; Lusso \& Risaliti 2016, see Lusso \& Risaliti 2017, for a physical interpretation), that 
is, the fractional disk contribution to the total emitted power increases for more luminous QSOs. Most previous works (e.g., Vignali et al. 2003; Steffen et al. 2006; Just et al. 2007; Jin et al. 2012; Marchese et al. 2012; Lusso \& Risaliti 2016; Nanni et al. 2017) found no evidence for evolution with redshift of $\alpha_{\mathrm{ox}}$. Recently, Risaliti \& Lusso (2019) exploit this apparent lack of evolution to propose QSOs as standard candles to infer cosmological parameters (see also Salvestrini et al. 2019).

Currently, $\approx 200$ QSOs have been discovered at $z \geq 6$, corresponding to about the first Gyr of the Universe (Bañados et al. 2016, and references therein; Mazzucchelli et al. 2017; Reed et al. 2017, 2019; Tang et al. 2017; Wang et al. 2017, 2018a,b; Chehade et al. 2018; Matsuoka et al. 2018a,b, 2019; Yang et al. 2019; Fan et al. 2019; Pons et al. 2019), with ULAS J1342+0928 holding the redshift record of $z=7.54$ (Bañados et al. 2018a). These rare QSOs were selected in wide-field optical/near-IR surveys such as SDSS, CFHQS, UKIDSS, Pan-STARRS1, ATLAS, and VIKING, and represent the extreme tail of the underlying SMBH population at early epochs. For instance, most of the known $z>6$ QSOs are extremely luminous ( $\left.\log L_{\mathrm{bol}} / L_{\odot} \approx 12-14\right)$ and massive (up to $\approx 10^{10} M_{\odot}$; Wu et al. 2015). The very existence of such massive black holes (BHs) in the early universe challenges our theoretical knowledge of SMBH formation and early growth (e.g., Woods et al. 2019, and references therein). In particular, in order to match the observed masses at $z \approx 6-7$, BH-seed models require extended periods of (possibly obscured) Eddingtonlimited $^{1}$, or even super-Eddington accretion, during which the structure and physics of the accretion may be different than at lower redshift, where QSOs are typically characterized by somewhat lower Eddington ratios (e.g., Shen \& Kelly 2012). This could produce a change in the $\alpha_{\mathrm{ox}}-L_{\mathrm{UV}}$ relation at high redshift.

Several works have compared the optical/UV continuum and emission-line properties (e.g., De Rosa et al. 2014; Shen et al. 2019) of QSOs at $z>6$ and at lower redshifts, generally finding a lack of evident evolution. However, the fraction of weak-line QSOs (WLQs, i.e., objects with C IV and Ly $\alpha+\mathrm{N}$ V rest-frame equivalent widths REW $<10 \AA$ and $\mathrm{REW}<15 \AA$, respectively; for instance Fan et al. 1999; Diamond-Stanic et al. 2009) has been suggested to increase toward high redshift (e.g., Luo et al. 2015; Bañados et al. 2016), in spite of the color selection used for $z>6$ QSOs that may be biased against objects with weak Ly $\alpha$ lines (Bañados et al. 2016). Since WLQs accrete preferentially with high Eddington ratios (e.g., Luo et al. 2015; Marlar et al. 2018), the higher fraction of WLQs may indicate that the known QSOs at $z>6$ generally accrete at higher Eddington ratios than at lower redshift, which is consistent with previous findings (e.g., Wu et al. 2015). Shen et al. (2019) find an excess of WLQs at $z>5.7$ compared to lower redshifts. Meyer et al. (2019) report a strong increase in the typical blueshift of the C IV emission line in QSOs at $z \gtrsim 6$, which can be linked again with the presence of a higher fraction of WLQs at high redshift (e.g., Luo et al. 2015). About half of the WLQ population is found to emit significantly weaker X-ray radiation than what is expected based on the UV luminosity (e.g., Ni et al. 2018). This is possibly linked to shielding by a geometrically thick inner accretion disk (e.g., Luo et al. 2015) as expected in the case of high Eddington-rate accretion.

$\mathrm{X}$-ray observations can provide useful insights into the accretion physics in an independent way and on smaller scales than those probed by optical/UV emission. For instance, in addition to the $\alpha_{\text {ox }}$ parameter (e.g., Lusso \& Risaliti 2017), the intrinsic

The Eddington luminosity is defined as $L_{\mathrm{Edd}}=1.26 \times 10^{38}\left(\frac{M_{\mathrm{BH}}}{M_{\odot}}\right)$. photon index $(\Gamma)$ of the hard X-ray power-law continuum carries information about the coupling between disk emission and the corona, and it is considered a proxy of the accretion rate. The relation between $\Gamma$ and the Eddington ratio has been established over a range of redshifts for sizable samples of sources: steeper slopes correspond to higher implied Eddington ratios (e.g., Shemmer et al. 2008; Risaliti et al. 2009; Brightman et al. 2013; Fanali et al. 2013, but see also Trakhtenbrot et al. 2017a).

Despite the large number of $z>6$ QSOs discovered to date, only $\approx 15$ (i.e., $\approx 8 \%$ of the known population at these redshifts) are currently covered by sensitive pointed or serendipitous $\mathrm{X}$-ray observations and only 11 are detected. This drastically limits our ability to use X-rays to investigate the accretion physics and structure in QSOs in the early universe. In this work, we present new Chandra observations for a sample of 10 QSOs at $z>6$. Along with archival data, we use these observations to constrain the X-ray properties of QSOs at $z>6$, derive the $\alpha_{\text {ox }}$ and $\Gamma$ parameters, and study possible dependencies upon redshift and luminosity. Our targets were selected to have virial estimates for $\mathrm{BH}$ masses from the $\mathrm{Mg}$ II emission line, allowing us to include Eddington ratios in our analysis. We adopt a flat cosmology with $H_{0}=67.7 \mathrm{~km} \mathrm{~s}^{-1}$ and $\Omega_{\mathrm{m}}=0.307$ (Planck Collaboration XIII 2016).

\section{The sample of $z>6$ QSOs}

\subsection{Targets of new $X$-ray observations}

We obtained Chandra observations of a sample of ten type 1 QSOs at $z=6.0-6.8$ (Tables 1 and 2), with virial estimates of $M_{\text {BH }}$ from near-IR spectroscopy (using the Mg II line ${ }^{2}$; e.g., Vestergaard \& Osmer 2009). The targets were selected to be radio-quiet or, at most, radio-moderate QSOs (see Sect. 2.3). Five of them have absolute magnitudes $-26.2<M_{1450 \AA}<-25.6$ (see red symbols in Fig. 1), close to the break luminosity regime of the QSO luminosity function at $z \approx 6$ (corresponding to $M_{1450 \AA} \approx-24.9$; Matsuoka et al. 2018c). This allows us to push the investigation of the X-ray emission of high-redshift QSOs down to a luminosity regime between typical SDSS QSOs (e.g., Pâris et al. 2018) and the fainter QSOs discovered by the SHELLQ survey (Matsuoka et al. 2016). This region of the QSO $L-z$ parameter space has been probed poorly to date at X-ray wavelengths. In fact, the only four $M_{1450 \AA}>-26$ QSOs at $z>6$ with previous X-ray data were serendipitously covered by X-ray observations (i.e., they were not targeted) and are not detected. Notably, with our new observations we more than triple the number of QSOs observed in X-rays at the highest redshifts $(z>6.5)$. The distributions of the absolute and apparent magnitudes at rest-frame $1450 \AA$ ( $M_{1450 \AA}$ and $m_{1450 \AA}$, respectively) as a function of redshift are shown in Fig. 1 (top and middle panels), and are compared with known $z>6$ QSOs not observed in the X-rays.

\subsection{Other $z>6$ QSOs observed in X-rays}

Nanni et al. (2017) studied the X-ray properties of all of the QSOs at $z>5.7$ previously covered by pointed or serendipitous X-ray observations, 14 of which are at $z>6$. We include in our analysis these $14 z>6$ QSOs. For these sources we used

\footnotetext{
2 Typical uncertainties for single-epoch mass estimates are $\gtrsim 0.5 \mathrm{dex}$ (e.g., Shen 2013, and references therein). In addition, the presence of spectral features (such as broad absorption lines) or weak emission lines can significantly affect the accuracy of the mass measurements.
} 
Table 1. Physical properties of $z>6$ QSOs with new or archival X-ray observations.

\begin{tabular}{|c|c|c|c|c|c|c|c|c|c|}
\hline $\begin{array}{l}\text { ID } \\
(1) \\
\end{array}$ & $\begin{array}{l}\text { RA } \\
(2)\end{array}$ & $\begin{array}{l}\text { Dec } \\
(3)\end{array}$ & $\begin{array}{c}z \\
(4) \\
\end{array}$ & $\begin{array}{c}M_{1450 \AA}\left(m_{1450 \AA}\right) \\
(5)\end{array}$ & $\begin{array}{c}\log \left(\frac{L_{\mathrm{bol}}}{L_{\odot}}\right) \\
(6)\end{array}$ & $\begin{array}{c}\log \left(\frac{M_{\mathrm{BH}}}{M_{\odot}}\right) \\
(7)\end{array}$ & $\begin{array}{c}\lambda_{\text {Edd }} \\
(8) \\
\end{array}$ & $\begin{array}{c}\text { Ref. (disc./z/M } M_{\mathrm{BH}} \text { ) } \\
(9)\end{array}$ & $\begin{array}{c}R \\
(10) \\
\end{array}$ \\
\hline \multicolumn{10}{|c|}{ New targets } \\
\hline CFHQS J0050+3445 & $00: 50: 06.67$ & $+34: 45: 21.65$ & 6.253 (Mg II) & $-26.70(20.11)$ & 13.45 & 9.41 & 0.34 & W10/W10/W10 & $<11.4$ \\
\hline VIK J0109-3047 & 01:09:53.13 & $-30: 47: 26.31$ & 6.7909 ([C II] $)$ & $-25.64(21.30)$ & 13.06 & 9.12 & 0.27 & V13/V16/M17 & $<34.1$ \\
\hline PSO J036+03 & 02:26:01.87 & $+03: 02: 59.42$ & $6.541([\mathrm{C} \mathrm{II}])$ & $-27.33(19.55)$ & 13.67 & 9.48 & 0.48 & V15/B15/M17 & $<2.1$ \\
\hline VIK J0305-3150 & 03:05:16.92 & $-31: 50: 55.9$ & 6.6145 ([C II] $)$ & $-26.18(20.72)$ & 13.26 & 8.95 & 0.63 & V13/V16/M17 & $<20.0$ \\
\hline SDSS J0842+1218 & $08: 42: 29.43$ & $+12: 18: 50.58$ & $6.0763([\mathrm{C} \mathrm{II}])^{(a)}$ & $-26.91(19.86)^{(a)}$ & 13.52 & 9.29 & 0.53 & $\mathrm{dR} 11 / \mathrm{D} 18 / \mathrm{dR} 11^{(*),(a)}$ & $<1.3$ \\
\hline PSO J167-13 & $11: 10: 33.98$ & $-13: 29: 45.60$ & $6.5148\left([\mathrm{C} \text { II] })^{(b)}\right.$ & $-25.57(21.25)$ & 13.03 & 8.48 & 1.11 & V15/M17/M17 & $<34.3$ \\
\hline CFHQS J1509-1749 & $15: 09: 41.78$ & $-17: 49: 26.80$ & $6.1225([\mathrm{C} \mathrm{II}])^{(a)}$ & $-27.14(19.64)^{(a)}$ & 13.61 & 9.47 & 0.42 & W07/D18/W10 ${ }^{(a)}$ & $<1.2$ \\
\hline CFHQS J1641+3755 & $16: 41: 21.73$ & $+37: 55: 20.15$ & 6.047 (Mg II) & $-25.67(21.09)$ & 13.07 & 8.38 & 1.51 & W07/W10/W10 & $<10.5$ \\
\hline PSO J338+29 & $22: 32: 55.14$ & $+29: 30: 32.31$ & $6.666([\mathrm{C} \mathrm{II}])$ & $-26.14(20.78)$ & 13.24 & 9.43 & 0.20 & V15/M17/M17 & $<21.0$ \\
\hline SDSS J2310+1855 & $23: 10: 38.89$ & $+18: 55: 19.93$ & $6.0031([\mathrm{C} \mathrm{II}])$ & $-27.80(18.95)$ & 13.85 & 9.62 & 0.52 & Wa13/Wa13/J16 & $<3.9$ \\
\hline \multicolumn{10}{|c|}{ QSOs with previous X-ray data } \\
\hline SDSS J0100+2802 & 01:00:13.02 & $+28: 02: 25.92$ & $6.3258([\mathrm{C} \mathrm{II}])$ & $-29.14(17.69)$ & 14.33 & 10.03 & 0.62 & Wu15/Wa16/Wu15 (*) & $<1.2$ \\
\hline ATLAS J0142-3327 & $01: 42: 43.73$ & $-33: 27: 45.47$ & $6.379([\mathrm{C} \mathrm{II}])^{(a)}$ & $-27.82(19.02)^{(a)}$ & 13.85 & - & - & C15/D18/- & $<4.2$ \\
\hline CFHQS J0210-0456 & 02:10:13.19 & $-04: 56: 20.90$ & $6.4323([\mathrm{C} \mathrm{II}])$ & $-24.53(22.33)$ & 12.65 & 7.90 & 1.76 & W10/W13/W10 & $<28.1$ \\
\hline CFHQS J0216-0455 & $02: 16: 27.81$ & $-04: 55: 34.10$ & $6.01(\operatorname{Ly} \alpha)$ & $-22.49(24.27)$ & 11.91 & - & - & W09/W09/- & $<23.1$ \\
\hline SDSS J0303-0019 & 03:03:31.40 & $-00: 19: 12.90$ & 6.078 (Mg II) & $-25.56(21.21)$ & 13.03 & 8.61 & 0.81 & $\mathrm{~J} 08 / \mathrm{K} 09 / \mathrm{dR} 11^{(*)}$ & $<11.4$ \\
\hline SDSS J1030+0524 & $10: 30: 27.11$ & $+05: 24: 55.06$ & 6.308 (Mg II) & $-26.99(19.84)$ & 13.55 & 9.21 & 0.68 & $\mathrm{~F} 01 / \mathrm{K} 07 / \mathrm{dR} 11^{(*)}$ & $<1.5$ \\
\hline SDSS J1048+4637 ${ }^{(c)}$ & $10: 48: 45.07$ & $+46: 37: 18.55$ & $6.2284(\mathrm{CO} 6-5)$ & $-27.24(19.57)$ & 13.64 & 9.55 & 0.38 & F03/Wa10/dR11 ${ }^{(*)}$ & $<0.5$ \\
\hline ULAS J1120+0641 & $11: 20: 01.48$ & $+06: 41: 24.30$ & $7.0842([\mathrm{C} \mathrm{II}])$ & $-26.63(20.38)$ & 13.42 & 9.39 & 0.33 & M11/V12/M17 & $<0.7$ \\
\hline SDSS J1148+5251 & $11: 48: 16.65$ & $52: 51: 50.39$ & $6.4189(\mathrm{CO} 6-5)$ & $-27.62(19.24)$ & 13.78 & 9.71 & 0.36 & F03/Wa11/dR11 (*) & $0.7_{-0.2}^{+0.2}$ \\
\hline SDSS J1306+0356 & 13:06:08.27 & $+03: 56: 26.36$ & 6.0337 ([C II] $)^{(a)}$ & $-26.82(19.94)^{(a)}$ & 13.49 & 9.30 & 0.48 & $\mathrm{~F} 01 / \mathrm{D} 18 / \mathrm{dR} 11^{(*),(a)}$ & $<1.5$ \\
\hline ULAS J1342+0928 & $13: 42: 08.27$ & $+09: 28: 38.61$ & 7.5413 ([C II] $)$ & $-26.76(20.34)$ & 13.47 & 8.89 & 1.14 & B18a/V17/B18a & $<4.7$ \\
\hline SDSS J1602+4228 & $16: 02: 53.98$ & $+42: 28: 24.94$ & $6.09(\operatorname{Ly} \alpha)$ & $-26.94(19.83)$ & 13.53 & - & - & F04/F04/- & $0.8_{-0.2}^{+0.2}$ \\
\hline SDSS J1623+3112 & $16: 23: 31.81$ & $+31: 12: 00.53$ & $6.26([\mathrm{C} \mathrm{II}])$ & $-26.55(20.27)$ & 13.39 & 9.15 & 0.54 & F04/Wa11/dR11 ${ }^{(*)}$ & $<2.3$ \\
\hline SDSS J1630+4012 & $16: 30: 33.90$ & $+40: 12: 09.69$ & 6.065 (Mg II) & $-26.19(20.58)$ & 13.26 & 8.96 & 0.62 & F03/I04/dR11 ${ }^{(*)}$ & $<2.2$ \\
\hline HSC J2216-0016 ${ }^{(c)}$ & $22: 16: 44.47$ & $-00: 16: 50.10$ & $6.10(\operatorname{Ly} \alpha)$ & $-23.62(23.16)$ & 12.32 & - & - & M16/M16/- & $<40.9$ \\
\hline
\end{tabular}

Notes. (1): QSO ID. (2) and (3): RA and Dec (J2000) from Bañados et al. (2016, 2018a). (4): Redshift and emission line from which it is derived. In cases of different values derived from different emission lines for the same source, we preferred the redshift derived from the [C II] line rather than the Mg II line, as discussed in Sect. 2.3. (5): Absolute and apparent magnitude at $1450 \AA$. $M_{1450 \AA}$ can vary by up to $\approx 0.3$ mag among different papers, depending on the prescription used to compute it (e.g., Omont et al. 2013 vs. Bañados et al. 2016 for CFHQS J1641+3755). We consistently assumed the values reported by Bañados et al. (2016) and Mazzucchelli et al. (2017), which used the same prescription, for all our sources. (6): Bolometric luminosity estimated from $M_{1450 \AA}$, using the bolometric correction of Venemans et al. (2016). (7): Virial black-hole mass estimated from the Mg II emission line. We note that Trakhtenbrot et al. (2017b) used different calibrations for the black-hole masses of several QSOs included in our sample, typically resulting in larger values (up to $\approx 0.2-0.3$ dex). (8): Eddington ratio: $\lambda_{\text {Edd }}=L_{\text {bol }} / L_{\text {Edd }}$. (10): Radio-loudness parameter (see Sect. 2.3) ${ }^{(*)}$ For these QSOs, black-hole masses have been modified according to the Vestergaard \& Osmer (2009) calibration, to be consistent with the other QSOs. In these cases, the references indicate the papers from which FWHM(Mg II) and $L_{3000 \AA}$ are collected. ${ }^{(a)}$ For these sources we updated magnitudes and black-hole masses according to the new [C II]-based redshifts provided by Decarli et al. (2018). These values differ negligibly from those derived assuming previous redshifts based on Mg II or Ly $\alpha$ emission lines. ${ }^{(b)}$ Willott et al. (2017) independently reported a slightly different value $(z=6.5157)$ from [C II]. ${ }^{(c)}$ Broad absorption-line QSOs (see Fan et al. 2004; Matsuoka et al. 2016).

References. For the QSO discovery, adopted redshift, and black-hole mass. B15: Bañados et al. (2015a); B18a: Bañados et al. (2018a); C15: Carnall et al. (2015); dR11: De Rosa et al. (2011); D18: Decarli et al. (2018); F01: Fan et al. (2001); F03: Fan et al. (2003); F04: Fan et al. (2004); I04: Iwamuro et al. (2004); J08: Jiang et al. (2008); J16: Jiang et al. (2016); K07: Kurk et al. (2007); K09: Kurk et al. (2009); M11: Mortlock et al. (2011); M16: Matsuoka et al. (2016); M17: Mazzucchelli et al. (2017); V12: Venemans et al. (2012); V13: Venemans et al. (2013); V15: Venemans et al. (2015); V16: Venemans et al. (2016); V17: Venemans et al. (2017); W07: Willott et al. (2007); W09: Willott et al. (2009); W10: Willott et al. (2010); W13: Willott et al. (2013); Wa10: Wang et al. (2010); Wa11: Wang et al. (2011); Wa13: Wang et al. (2013); Wa16: Wang et al. (2016); Wu15: Wu et al. (2015).

the magnitudes at $1450 \AA$ provided by Bañados et al. (2016). We also include ULAS J1342+0928, which was discovered after the Nanni et al. (2017) work, and whose X-ray properties, magnitudes, and black-hole mass have been presented by Bañados et al. (2018a,b). We thus include in our analysis a total of $15 z>6$ QSOs with sensitive ${ }^{3}$ archival observations in the $\mathrm{X}$-ray band.

Seven of these QSOs were observed by Chandra only, three by XMM-Newton only, four by both Chandra and XMMNewton, and one by Swift. Recently SDSS J1030+0524 has been the target of a long Chandra imaging campaign $(\approx 480 \mathrm{ks}$, Nanni et al. 2018), and was previously observed with both

3 We do not consider very shallow X-ray surveys, like the ROSAT AllSky survey, which would provide only very loose upper limits on the X-ray fluxes of high-redshift QSOs.
Chandra (with a shallow 8 ks observation; Brandt et al. 2002) and XMM-Newton (75 ks after background filtering; Farrah et al. 2004). However, considering the long separation between the old and new observations $(\approx 10-15$ years in the observed frame), and the hints for strong variation affecting its flux during this timespan, as discussed in Nanni et al. (2018), we limited our analysis to the deep 2017 Chandra dataset. Similarly, we consider only the $\approx 80 \mathrm{ks}$ Chandra observation of SDSS J1148+5251 (Gallerani et al. 2017), and discarded a 2004 XMM-Newton observation with a nominal exposure time of $\approx 26 \mathrm{ks}$, which is however almost completely affected by background flaring. As a result, for nine QSOs out of the 15 objects with archival observations we used only Chandra data, for 3 QSOs we used only XMM-Newton data, for 2 QSOs we used data from both observatories, and for one we used Swift data (see Table 2). 
Table 2. Summary of our new Chandra and archival X-ray observations of $z>6$ QSOs.

\begin{tabular}{|c|c|c|c|}
\hline ID & OBSID & Date & $T_{\exp }[\mathrm{ks}]$ \\
\hline \multicolumn{4}{|c|}{ New observations } \\
\hline CFHQS J0050+3445 C & 20393 & $2017-09-25$ & 33.5 \\
\hline VIK J0109-3047C & 20398 & 2019-05-07 & 37.0 \\
\hline "C & 22214 & 2019-05-10 & 29.5 \\
\hline PSO J036+03 ${ }^{\mathrm{C}}$ & 20390 & 2018-10-09 & 25.9 \\
\hline VIK J0305-3150 C & 20394 & $2018-05-11$ & 49.9 \\
\hline SDSS J0842+1218 ${ }^{\mathrm{C}}$ & 20392 & 2018-01-01 & 28.7 \\
\hline PSO J167-13 ${ }^{\mathrm{C}}$ & 20397 & $2018-02-20$ & 59.3 \\
\hline CFHQS J1509-1749C & 20391 & 2018-06-06 & 26.8 \\
\hline CFHQS J1641+3755C & 20396 & $2018-11-15$ & 20.8 \\
\hline "C & 21961 & $2018-11-17$ & 33.5 \\
\hline PSO J338+29C & 20395 & $2018-01-30$ & 54.2 \\
\hline SDSS J $2310+1855^{\mathrm{C}}$ & 20398 & 2017-09-30 & 17.9 \\
\hline \multicolumn{4}{|c|}{ Archival observations } \\
\hline SDSS J0100+2802 & 17087 & $2015-10-16$ & 14.8 \\
\hline$" \mathrm{X}$ & 0790180701 & 2016-06-29 & $44.9 / 60.7 / 60.4$ \\
\hline ATLAS J0142-3327s & 00290624001 & $2007-09-11$ & 20.9 \\
\hline CFHQS J0210-0456 & 0677630133 & $2012-07-10$ & $8.0 / 10.6 / 10.6$ \\
\hline$" \mathrm{X}$ & 0677640133 & $2012-01-12$ & $8.4 / 10.6 / 10.5$ \\
\hline CFHQS J0216-0455 & 0112370601 & $2002-08-12$ & $29.4 / 37.9 / 37.9$ \\
\hline SDSS J0303-0019C & 13349 & 2011-11-27 & 1.5 \\
\hline SDSS J1030+0524 & 18185 & $2017-01-17$ & 46.3 \\
\hline "C & 18186 & $2017-01-25$ & 34.6 \\
\hline "C & 18187 & $2017-03-22$ & 40.4 \\
\hline "C & 19926 & $2017-05-25$ & 49.4 \\
\hline "C & 19987 & 2017-01-18 & 126.4 \\
\hline "C & 19994 & $2017-01-27$ & 32.7 \\
\hline "C & 19995 & $2017-01-27$ & 26.7 \\
\hline "C & 20045 & $2017-03-24$ & 61.3 \\
\hline "C & 20046 & $2017-03-26$ & 36.6 \\
\hline "C & 20081 & $2017-05-27$ & 24.9 \\
\hline SDSS J1048+4637 ${ }^{\mathrm{C}}$ & 5608 & $2005-01-10$ & 15.0 \\
\hline ULAS J1120+0641 ${ }^{\mathrm{C}}$ & 13203 & 2011-02-04 & 15.8 \\
\hline "X & 0693990101 & $2012-05-23$ & $24.1 / 46.5 / 45.8$ \\
\hline "X & 0693990201 & 2012-06-18 & $71.9 / 108.0 / 108.1$ \\
\hline "X & 0693990301 & 2012-06-20 & $56.4 / 83.6 / 84.1$ \\
\hline SDSS J1148+5251 ${ }^{\mathrm{C}}$ & 17127 & 2015-09-02 & 77.8 \\
\hline SDSS J1306+0356 ${ }^{\mathrm{C}}$ & 3358 & 2002-01-29 & 8.2 \\
\hline "C & 3966 & $2003-11-29$ & 118.2 \\
\hline ULAS J1342+0928 C & 20124 & $2017-12-15$ & 24.7 \\
\hline "C & 20887 & $2017-12-17$ & 20.4 \\
\hline SDSS J1602+4228 & 5609 & $2005-10-29$ & 13.2 \\
\hline SDSS J1623+3112 & 5607 & $2004-12-29$ & 17.2 \\
\hline SDSS J1630+4012 & 5618 & 2005-11-04 & 27.4 \\
\hline HSC J2216-0016 & 0673000145 & $2011-12-08$ & $3.7 / 4.2 / 4.2$ \\
\hline
\end{tabular}

Notes. C - source observed with Chandra. X - source observed with XMM-Newton. Exposure times are filtered for background flaring and correspond to the PN, MOS1, and MOS2 cameras, respectively. $\mathrm{S}$ - source observed with Swift.

We searched the literature to retrieve black-hole mass estimates for these 15 QSOs (see Table 1). Since different authors used different calibrations to obtain estimates of blackhole masses, we recalibrate the values found in the literature to match the calibration of Vestergaard \& Osmer (2009), as marked in Table 1. We also modified luminosities and masses for our chosen cosmology. Furthermore, for consistency, we applied the same X-ray analysis (see Sect. 3) to these archival observations.

\subsection{General properties of the sample}

The main physical properties of our sample are reported in Table 1. For many of our targets, slightly different redshift values are reported in the literature, derived from the $\operatorname{Mg}$ II (2799 $\AA$ ) and [C II] $(158 \mu \mathrm{m})$ emission lines. When a [C II] measurement is available, we adopt it since the [C II] line is considered a better indicator of the systemic redshift than the $\mathrm{Mg}$ II line (e.g., Decarli et al. 2018), which sometimes displays significant blueshifts in the observed wavelength (e.g., Plotkin et al. 2015; Shen et al. 2016), possibly due to outflowing material in the broad emission-line region (e.g., $\approx 1700 \mathrm{~km} \mathrm{~s}^{-1}$ for SDSS J0109-3047, corresponding to $\Delta z \approx 0.04$; Venemans et al. 2016).

We computed the bolometric luminosities $\left(L_{\mathrm{bol}}\right)$ consistently for all our targets using the bolometric correction of Venemans et al. (2016), which was also used in Decarli et al. (2018): $\log \left(\frac{L_{\text {bol }}}{\operatorname{erg~s}^{-1}}\right)=4.553+0.911 \times \log \left(\frac{\lambda L_{\lambda}(1450 \AA)}{\operatorname{erg~s}^{-1}}\right)$. The typical uncertainty on $L_{\text {bol }}$ derived with this relation is $\sim 7 \%$. We thus provide homogeneously derived $L_{\text {bol }}$ rather than compiling values found in the literature, which are derived using different indicators of the bolometric luminosity (i.e., $L_{3000 \AA}$ and $M_{1450 \AA}$ ) and different bolometric corrections.

None of the QSOs included in our sample has been detected in the FIRST (which covers 16 of the 25 QSOs in our sample; Becker et al. 1995) or NVSS (covering all of our $z>6$ QSOs; Condon et al. 1998) radio surveys. We report in Table 1 the radio-loudness parameter $R=f_{v, 5 \mathrm{GHz}} / f_{v, 4400 \AA}$ (Kellermann et al. 1989), i.e., the ratio of the flux densities at rest-frame $5 \mathrm{GHz}$ and $4400 \AA$, or its upper limit, for the QSOs in our sample. $R$ values of QSOs included in the compilation of Bañados et al. (2015b) are taken from that work, including the only two QSOs detected at $1.4 \mathrm{GHz}$ (with $R=0.7-0.8$ ). For the remaining sources, we derived $f_{v, 4400 \AA}$ from $m_{1450 \AA}$ (Col. 5 of Table 1), assuming a power-law continuum with $\alpha=-0.3$, following Bañados et al. (2016). Upper limits on the radio emission at $1.4 \mathrm{GHz}$ are derived as $3 \times \mathrm{rms}$ of the FIRST or NVSS surveys. For CFHQS J0216-0455, we used the rms of the VLA observations in the SXDS field (Simpson et al. 2006). Finally, we estimated the upper limits on $f_{v, 5 \mathrm{GHz}}$ assuming a power-law spectrum with $\alpha=-0.75$. Based on their upper limits on $R$, all of our sources are either radio-quiet $(R<10)$ or at most radiointermediate $(R<40)$. We thus do not expect their X-ray emission to be significantly affected by a jet-linked contribution (e.g., Miller et al. 2011). Bañados et al. (2015b) reported a radio-loud QSO fraction of $\approx 8 \%$ among the $z \approx 6$ population. The only three radio-loud QSOs at $z>6$ are not covered by X-ray observations and thus are not included in our sample.

It is difficult to establish firmly how many of the QSOs in the sample can be classified as WLQs, mainly because of the limited quality of the optical/UV spectra and spectral coverage. Beside the known WLQ SDSS J0100+2802 (Wu et al. 2015), other WLQ candidates are VIK J0109-3047, ULAS J1342+0928, and SDSS J2310+1855, all with REW(C IV) $\approx 10-15$ (see Table 1 for the spectral references). However, several of the sources lack measurements of REW(CIV). Furthermore, as reported in Table 1, two QSOs are classified as broad absorption-line QSOs (BALQSOs), which usually show weak X-ray emission as well (e.g., Gallagher et al. 2006; Gibson et al. 2009; Wu et al. 2010; Luo et al. 2014).

\section{Data analysis}

\subsection{X-ray data reduction}

Table 2 summarizes the basic information about the X-ray observations of our new targets and archival sources. We reprocessed the Chandra observations with the chandra_repro script 


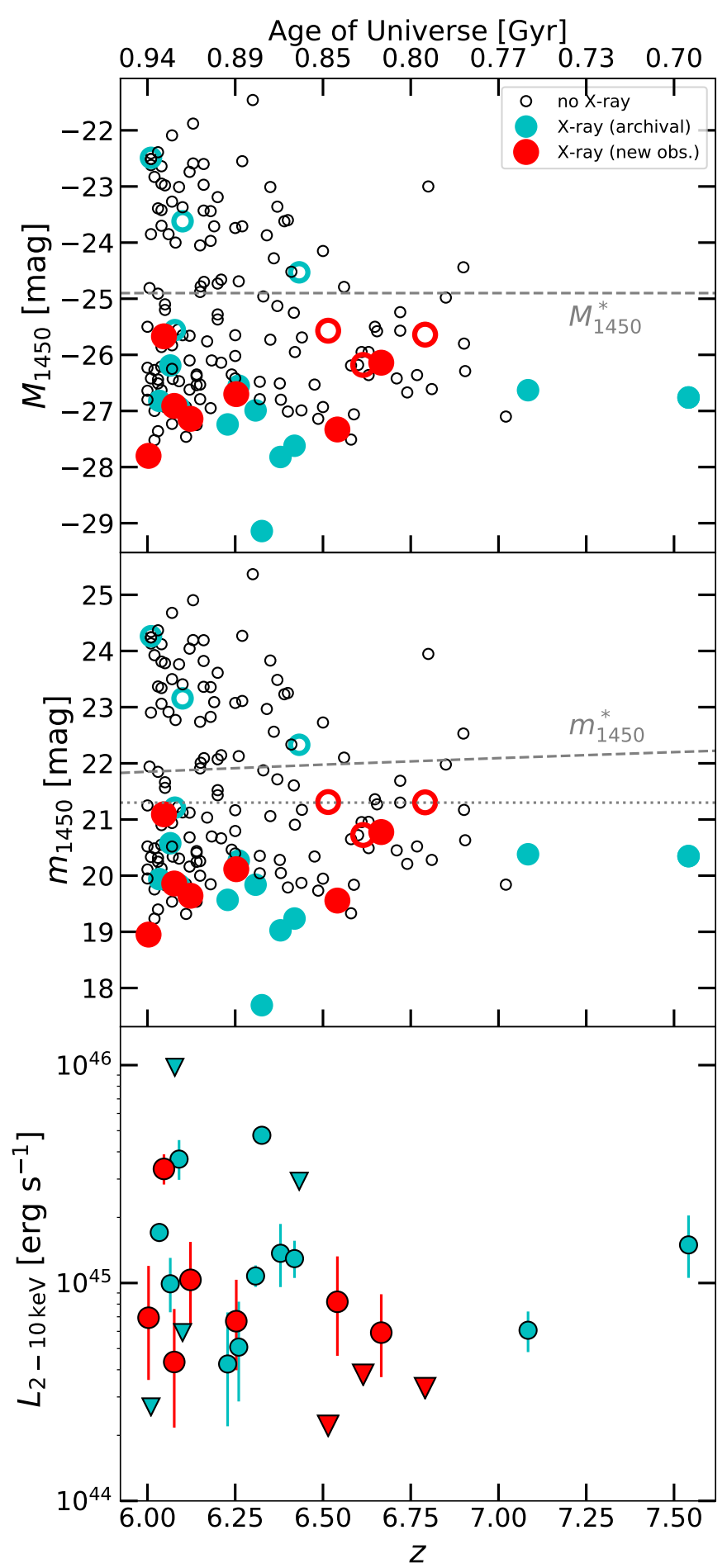

Fig. 1. Top and middle panels: distribution of $M_{1450 \AA}$ and $m_{1450 \AA}$ as function of redshift. Small black open circles are QSOs not covered by X-ray observations (Bañados et al. 2016; Mazzucchelli et al. 2017; Reed et al. 2017; Tang et al. 2017; Wang et al. 2017, 2018a,b; Chehade et al. 2018; Matsuoka et al. 2018b,a; Yang et al. 2019). Cyan symbols are QSOs with archival X-ray data (see Table 1). Red symbols are QSOs covered by the new X-ray observations presented in this work. Filled symbols are X-ray detected, open symbols are not detected. Dashed lines represent the break magnitudes of the QSO luminosity function (Matsuoka et al. 2018c). The dotted line represents our magnitude selection. Bottom panel: X-ray luminosity (derived as described in Sect. 3) as a function of redshift. Symbols are the same as above, but upper limits on X-ray luminosity for undetected QSOs are shown as downward-pointing triangles. in CIAO $4.10^{4}$, using CALDB $\mathrm{v} 4.8 .1^{5}$, setting the option check_vf_pha=yes in the case of observations taken in very faint mode. We created exposure maps with the fluximage script. Spectra, response matrices, and ancillary files for sources and associated background were extracted using the specextract tool.

SDSS J1030+0524 has been observed with ACIS-I in ten individual pointings over five months, for a total of $\approx 480 \mathrm{ks}$ (see Table 2 and Nanni et al. 2018). Similarly, VIK J0109-3047, SDSS J1306+0356, ULAS J1342+0928, and CFHQS J1641+3755 have been targeted with two Chandra observations, for a total of $\approx 65,126,45$, and $54 \mathrm{ks}$, respectively (see Table 2 and Bañados et al. 2018b). For these sources, we checked for astrometry issues and merged the individual observations with the reproject_obs tool, and derived merged images and exposure maps. In doing this, we effectively combine the different pointings into a single, longer exposure. Spectra, response matrices, and ancillary files extracted from the single pointings were added using the mathpha, addrmf, and addarf HEASOFT tools ${ }^{6}$, respectively, weighting by the individual exposure times.

$X M M-N e w t o n$ observations have been processed with SAS v16.1.0., following the standard procedure ${ }^{7}$ and filtering for periods of high background levels imposing count-rate thresholds of $<0.4$ and $<0.35 \mathrm{cts} \mathrm{s}^{-1}$ in the $10<E<12 \mathrm{keV}$ and $E>10 \mathrm{keV}$ bands for the EPIC/PN and EPIC/MOS cameras, respectively. We created images and exposure maps, and extracted spectra, response matrices, and ancillary files using the evselect, eexpmap, backscale, rmfgen, and arfgen tools.

In the case of sources targeted by multiple XMM-Newton pointings (i.e., CFHQS J0210-0456 and ULAS J1120+0641; see Table 2), we merged the different datasets for each EPIC camera with the merge tool, and, similarly to what we did for Chandra sources, we added the spectra extracted from each observation with the epicspeccombine tool. We also averaged the response matrices and ancillary files with the addrmf and addarf tools, weighting by the exposure times of the individual observations. We note that epicspeccombine returns as output a summed spectrum with exposure time set to the average value of the two input spectra, and the sum of the two input ancillary files. This is the equivalent of observing the source for half of the total time with a fictional camera with twice the sensitivity of the actual camera. By changing with dmhedit the exposure time keyword of the output summed spectrum to the summed exposure time of the two input spectra, and by computing the weighted average of the response matrices and ancillary files with addrmf and addarf, we return to the case in which the source is observed by the actual camera for a longer exposure time. The two cases are equivalent when spectra and ancillary files are used together (e.g., performing spectral analysis with XSPEC). However, in Sect. 3.3 we will use the ancillary files alone to compute the count-rate to flux conversion factors. In such a case, the use of the summed ancillary files obtained as output of epicspeccombine would not be correct.

We then used the merged images to compute source photometry (see Sect. 3.2). Since these sources were placed at similar off-axis angles in the different pointings, by merging the observations for each camera we effectively combine them into single

\footnotetext{
4 http://cxc.harvard.edu/ciao/

5 http://cxc.harvard.edu/caldb/

6 https://heasarc.gsfc.nasa.gov/docs/software/heasoft/

7 https://www. cosmos.esa.int/web/xmm-newton/

sas-threads
} 
and longer observations. However, we keep the different cameras separated, as the responses are significantly different. We then combined the scientific results, as described in Sect. 3.3.

We reduced Swift-XRT data for ATLAS J0142-3327 as in Nanni et al. (2017), using the standard software (HEADAS v. $6.18)^{8}$ and procedures ${ }^{9}$. An ancillary file has been extracted with the xrtmkarf tool.

\subsection{Detection procedure}

For Chandra observations, we used circular source extraction regions centered on the optical positions of the targets and with radii of 2 arcsec, to account for X-ray and optical positional uncertainties, and any possible small X-ray-to-optical offset. This region size encompasses $\approx 100 \%$ and $\approx 90 \%$ of the Chandra PSF at $E=1.5$ and $6.4 \mathrm{keV}$, respectively, for an on-axis position. The background levels are evaluated in local annular regions centered on the targets, with inner and outer radii of 4 and 24 arcsec, respectively, free of contaminating sources. All the sources in our sample covered by Chandra observations were observed on axis, except for SDSS J0303-0019, which is observed at an off-axis angle of $\approx 4.8$ arcmin.

For XMM-Newton observations, we used circular source extraction regions centered on the optical positions of the targets and with radii of $10-30$ arcsec (corresponding to $\approx 50-80 \%$ of the PSF), depending on the off-axis angle of the source (0-6 arcmin) and the presence of nearby detected objects that could contaminate the photometry. Circular background extraction regions are placed at nearby locations free of evident detected sources and have radii of 60-80 arcsec. For the SwiftXRT observation of ATLAS J0142-3327 we computed the source photometry in a circular region with radius 10 arcsec, which equates to $\approx 50 \%$ of the PSF (Moretti et al. 2005), and the background photometry in a nearby circular region with radius $\approx 72$ arcsec.

We ran the detection procedure in three energy bands $(0.5-2$, $2-7$, and $0.5-7 \mathrm{keV}$, which we refer to as the soft, hard, and full bands, respectively) separately for every available instrument (ACIS, EPIC/PN, EPIC/MOS1, EPIC/MOS2, and XRT). Different images of one object taken with the same instrument were merged, as described in Sect. 3.1. We computed the detection significance in each energy band using the binomial no-source probability (Weisskopf et al. 2007; Broos et al. 2007)

$P_{B}(X \geq S)=\sum_{X=S}^{N} \frac{N !}{X !(N-X) !} p^{X}(1-p)^{N-X}$,

where $S$ is the total number of counts in the source region in the considered energy band, $B$ is the total number of counts in the background region, $N=S+B$, and $p=1 /(1+$ BACKSCAL $)$, with BACKSCAL being the ratio of the background and source region areas. For sources observed by multiple instruments, we consider the quantity $P_{B}^{\mathrm{TOT}}=\prod_{i} P_{B}^{i}$ as the final binomial no-source probability in one energy band, where the product is performed over all the instruments used to observe a source. We consider a source to be detected if $\left(1-P_{B}\right)>0.99$. Out of the 111 analyzed images ( 25 objects in the three energy bands, some of which were observed by different instruments, see Table 3 ), we expect $111 \times P_{B} \approx 1$ false detection with the adopted significance threshold.

\footnotetext{
8 https://heasarc.gsfc.nasa.gov/docs/software/ lheasoft/

9 https://swift.gsfc.nasa.gov/analysis/
}

Figure 2 displays the X-ray images of our new targets in the three energy bands (see Nanni et al. 2017 and Bañados et al. 2018 b, for similar images for the archival sources). Detected and undetected sources are identified with green and red circles, respectively. Three of our 10 observed targets are detected in all of the three considered bands, four QSOs are detected in the soft and full bands only, and three are not detected in any band. Reasonably different sizes for the source and background extraction regions do not affect these results.

\subsection{Photometry, fluxes, and luminosities}

We computed the net counts and associated uncertainties (or upper limits in the case of non-detections) by deriving the probability distribution function of net counts with the method of Weisskopf et al. (2007, see their Appendix A3), which correctly accounts for the Poisson nature of both source and background counts. For sources detected by an instrument in one energy band, we report in Table 3 the nominal value of the net counts, corresponding to the peak of the probability distribution, and the errors corresponding to the narrowest $68 \%$ confidence interval. For undetected sources we report the upper limit corresponding to the $90 \%$ confidence interval. These values are not corrected for the fraction of PSF excluded in the extraction regions.

We used the probability distribution functions of the net counts in the soft and hard bands to constrain the hardness ratio HR $=(H-S) /(H+S)$, where $S$ and $H$ are the observed net counts in the soft and hard bands, respectively: we randomly picked a pair of values following such functions and computed HR. Repeating the procedure 10000 times, we constructed the probability distribution function of HR, and computed the $68 \%$ confidence interval, or $90 \%$ upper limit in the case of sources undetected in the hard band (Table 3 ). We found no significantly different hardness-ratio values using the Bayesian Estimation of Hardness Ratios (BEHR) code (Park et al. 2006). The last column of Table 3 reports the effective photon indices corresponding to the HR values, computed assuming a power law model and Galactic absorption (Kalberla et al. 2005), and accounting for the effective area of each instrument at the time of each observation and at the position of each source on the detector.

The probability distribution functions of X-ray flux in the three energy bands have been derived from the net count-rate probability distribution function assuming a power-law spectrum with $\Gamma=2.0$ (typical of luminous QSOs, e.g., Shemmer et al. 2006a; Nanni et al. 2017, see also Sect. 4.3), accounting for Galactic absorption (Kalberla et al. 2005) and using the response matrices and ancillary files extracted at the position of each target. All of the ancillary files are corrected for the fraction of the PSF not included in the extraction regions. Thus, fluxes and derived quantities are corrected for PSF effects. Table 4 reports the fluxes corresponding to the peak of the probability distribution functions, and the uncertainties corresponding to the narrowest interval containing $68 \%$ of the total probability for sources detected in an energy band. For undetected objects we report the upper limit corresponding to the $90 \%$ probability.

For QSOs observed by different instruments, we derived the flux probability distribution function for each instrument, multiplied them together and then renormalized the result to obtain the average distribution. This was used to compute the nominal fluxes and uncertainties. Deep observations produce narrower probability distribution functions than shallower pointings, and thus dominate the averaged final distribution. This averaging procedure works if a source did not vary strongly between the different observations; otherwise, the flux probability 

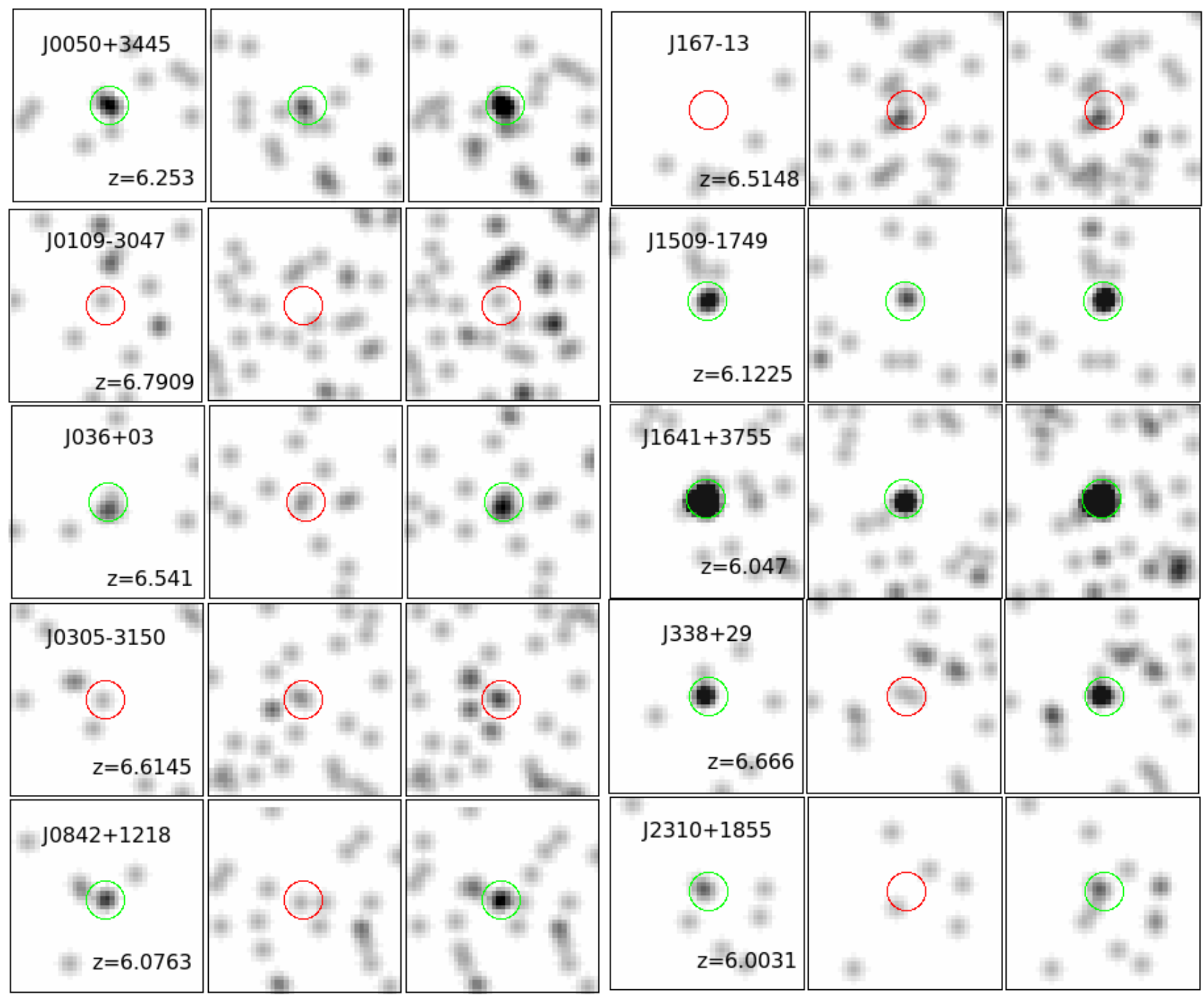

Fig. 2. Smoothed Chandra images $\left(40 \times 40\right.$ pixels; i.e., $\left.\approx 20^{\prime \prime} \times 20^{\prime \prime}\right)$ of our ten new targets (rows, as annotated) in soft (first column), hard (second column), and full (third column) band. Circles represent the source extraction regions $(R=2 \mathrm{arcsec})$ centered on the optical positions of the targets, and used to compute source photometry. Green and red circles are used for detected and undetected sources, respectively.

distribution functions for the individual instruments do not overlap and their product is null. There is no such case in our sample. We note, however, that for some objects observed by multiple instruments several months apart (e.g., SDSS J0100+2802 and ULAS J1120+0641), the flux probability distribution functions of the individual instruments are slightly shifted, although they still largely overlap. While this shift can be simply explained by statistical fluctuations of the measured counts, we cannot exclude some level of source variability. In this case our results would correspond to fluxes averaged over the different observed states. We note that Shemmer et al. (2017) report no significant evolution of QSO X-ray variability amplitude with redshift, at least up to $z \approx 4.3$.

Luminosities in the rest-frame $2-10 \mathrm{keV}$ band (Table 4 ) and monochromatic luminosities at $2 \mathrm{keV}$ have been computed from the unabsorbed (i.e., corrected for Galactic absorption) fluxes in the soft band, assuming again $\Gamma=2.0$. Figure 1 (bottom panel) presents the distribution of X-ray luminosity vs. redshift for $z \geq$ 6 QSOs. A short extrapolation is needed in the X-ray luminosity calculation, since the emission at rest-frame $2 \mathrm{keV}$ is redshifted below $0.5 \mathrm{keV}$ at $z>6$, and is thus not directly probed by X-ray observations.

\section{Results}

\section{1. $\alpha_{o x}$ vs. Iuminosity, redshift, and QSO properties}

We computed $L_{2500 \AA}$ from the $1450 \AA$ magnitude assuming a power-law spectrum with $\alpha=-0.3$, (e.g., Bañados et al. 2016; Selsing et al. 2016). Table 4 shows the $\alpha_{\text {ox }}$ values for the sources in our sample. The reported errors account only for the errors on the X-ray photometry, which dominate over the uncertainties on $L_{2500 \AA}$. Errors on the UV luminosities are dominated by the assumed UV spectral slope rather than measurement errors. For instance, assuming $\alpha=-0.5$ (e.g., Vanden Berk et al. 2001) returns $\alpha_{\text {ox }}$ values steeper by $\approx 0.02$ than the reported ones, and thus still well within the errors on $\alpha_{\text {ox }}$ reported in Table 4.

We plotted in Fig. $3 \alpha_{\text {ox }}$ vs. UV luminosity for our sample, and compared them with the best-fit relations of Just et al. (2007), Lusso \& Risaliti (2016), and Martocchia et al. (2017). All of these relations are very similar in the luminosity regime probed by our sources. We plot as small black symbols the sample of $z<6$ QSOs (from Shemmer et al. 2006b; Steffen et al. 2006, and Just et al. 2007) used to fit the Just et al. (2007) relation. We also show the sample of $>2000$ QSOs of Lusso \& Risaliti (2016) as a color-coded map based on the 
Table 3. Observed X-ray photometry and hardness ratios.

\begin{tabular}{|c|c|c|c|c|c|}
\hline \multirow[t]{2}{*}{ ID } & \multicolumn{3}{|c|}{ Netcounts } & \multirow[t]{2}{*}{ HR } & \multirow[t]{2}{*}{$\Gamma_{\text {eff }}$} \\
\hline & SB & $\mathrm{HB}$ & FB & & \\
\hline \multicolumn{6}{|c|}{ New observations } \\
\hline CFHQS J0050+3445 & $4.8_{-1.9}^{+2.6}$ & $2.7_{-1.4}^{+2.1}$ & $7.4_{-2.5}^{+3.2}$ & $-0.26_{-0.39}^{+0.26}$ & $1.68_{-0.28}^{+1.10}$ \\
\hline VIK J0109-3047 & $<3.5$ & $<2.3^{-1 .}$ & $<3.1$ & - & - \\
\hline PSO J036+03 & $3.9_{-1.7}^{+2.4}$ & $<4.9$ & $5.5_{-2.1}^{+2.8}$ & $<0.02$ & $>1.12$ \\
\hline VIK J0305-3150 & $<3.6$ & $<4.7$ & $<5.7$ & - & - \\
\hline SDSS J0842+1218 & $2.8_{-1.4}^{+2.1}$ & $<3.5$ & $3.3_{-1.7}^{+2.4}$ & $<0.17$ & $>0.77$ \\
\hline PSO J167-13 & $<2.3^{-1.4}$ & $<7.2$ & $<6.8$ & - & - \\
\hline CFHQS J1509-1749 & $5.7_{-2.1}^{+2.8}$ & $2.6_{-1.4}^{+2.1}$ & $8.4_{-2.7}^{+3.4}$ & $-0.33_{-0.35}^{+0.26}$ & $1.94_{-0.60}^{+1.06}$ \\
\hline CFHQS J1641+3755 & $39.5_{-6.0}^{+6.6}$ & $8.3_{-2.7}^{+3.4}$ & $47.8_{-6.7}^{+7.3}$ & $-0.65_{-0.15}^{+0.08}$ & $2.15_{-0.19}^{+0.49}$ \\
\hline PSO J338+29 & $5.6_{-2.1}^{+2.8}$ & $<4.7$ & $6.9_{-2.5}^{+3.2}$ & $<-0.06$ & $>1.30$ \\
\hline SDSS J2310+1855 & $2.9_{-1.4}^{+2.1}$ & $<3.7$ & $3.7_{-1.7}^{+2.4}$ & $<0.18$ & $>0.74$ \\
\hline \multicolumn{6}{|c|}{ Archival observations } \\
\hline SDSS J0100+2802 (Chandra) & $12.8_{-3.3}^{+4.0}$ & $<5.1$ & $14.6_{-3.6}^{+4.2}$ & $<-0.42$ & $>1.88$ \\
\hline " (PN) & $149.5_{-13.6}^{+14.3}$ & $31.2_{-8.4}^{+9.0}$ & $180.7_{-16.1}^{+16.8}$ & $-0.66_{-0.11}^{+0.07}$ & $2.18_{-0.20}^{+0.38}$ \\
\hline " (MOS1) & $74.6_{-9.1}^{+9.7}$ & $<11.3$ & $78.6_{-10.0}^{+10.6}$ & $<-0.72$ & $>2.68$ \\
\hline " (MOS2) & $52.3_{-7.8}^{+8.4}$ & $11.4_{-4.5}^{+5.2}$ & $64.0_{-9.1}^{+9.8}$ & $-0.64_{-0.16}^{+0.11}$ & $2.44_{-0.33}^{+0.62}$ \\
\hline ATLAS J0142-3327 (Swift) & $11.0_{-3.3}^{+4.0}$ & $<4.2$ & $11.5_{-3.5}^{+4.3}$ & $<-0.41$ & $>1.68$ \\
\hline CFHQS J0210-0456 (PN) & $<14.2$ & $<18.4$ & $<12.3$ & - & - \\
\hline " (MOS1) & $<11.2$ & $<4.5$ & $<7.1$ & - & - \\
\hline " (MOS2) & $<17.3$ & $<14.2$ & $<13.5$ & - & - \\
\hline CFHQSJ0216-0455 (PN) & $<9.3$ & $<4.0$ & $<7.2$ & - & - \\
\hline " (MOS1) & $<8.5$ & $<7.0$ & $<11.5$ & - & - \\
\hline "(MOS2) & $<3.4$ & $<3.4$ & $<3.7$ & - & - \\
\hline SDSS J0303-0019 (Chandra) & $<2.3$ & $<3.9$ & $<3.9$ & - & - \\
\hline SDSS J1030+0524 (Chandra) & $78.2_{-8.6}^{+9.2}$ & $46.4_{-6.7}^{+7.4}$ & $124.6_{-11.0}^{+11.7}$ & $-0.26_{-0.11}^{+0.07}$ & $1.91_{-0.17}^{+0.25}$ \\
\hline SDSS J1048+4637 (Chandra) & $2.9_{-1.4}^{+2.1}$ & $<2.3$ & $2.8_{-1.4}^{+2.1}$ & $<-0.02$ & $>0.66$ \\
\hline ULAS J1120+0641 (Chandra) & $3.9_{-1.7}^{+2.4}$ & $<5.1$ & $5.7_{-2.1}^{+2.8}$ & $<0.19$ & $>0.39$ \\
\hline " (PN) & $21.2_{-8.3}^{+8.9}$ & $<10.7$ & $<32.3$ & $<-0.21$ & $>1.39$ \\
\hline " (MOS1) & $14.9_{-6.4}^{+7.0}$ & $<6.2$ & $<19.2$ & $<-0.24$ & $>1.41$ \\
\hline " (MOS2) & $17.8_{-6.3}^{+7.0}$ & $<24.2$ & $32.3_{-9.1}^{+9.7}$ & $<0.25$ & $>0.47$ \\
\hline SDSS J1148+5251 (Chandra) & $26.5_{-4.9}^{+5.5}$ & $10.2_{-3.0}^{+3.7}$ & $36.7_{-5.8}^{+6.5}$ & $-0.44_{-0.18}^{+0.12}$ & $1.87_{-0.29}^{+0.50}$ \\
\hline SDSSJ1306+0356 (Chandra) & $105.0_{-9.9}^{+10.6}$ & $28.0_{-5.0}^{+5.7}$ & $133.1_{-5.0}^{+5.7}$ & $-0.57_{-0.10}^{+0.05}$ & $1.78_{-0.14}^{+0.28}$ \\
\hline ULASJ1342+0928 (Chandra) & $9.7_{-2.9}^{+3.5}$ & $4.4_{-1.9}^{+2.7}$ & $14.1_{-3.6}^{+4.2}$ & $-0.36_{-0.29}^{+0.20}$ & $1.88_{-0.44}^{+0.86}$ \\
\hline SDSSJ1602+4228 (Chandra) & $22.9_{-4.5}^{+5.1}$ & $3.7_{-1.7}^{+2.4}$ & $25.6_{-4.9}^{+5.5}$ & $-0.70_{-0.14}^{+0.13}$ & $2.21_{-0.39}^{+0.63}$ \\
\hline SDSSJ1623+3112 (Chandra) & $3.9_{-1.7}^{+2.4}$ & $2.9_{-1.4}^{+2.1}$ & $6.8_{-2.3}^{+3.0}$ & $-0.14_{-0.36}^{+0.31}$ & $0.89_{-0.58}^{+0.74}$ \\
\hline SDSSJ1630+4012 (Chandra) & $12.7_{-3.3}^{+4.0}$ & $4.8_{-1.9}^{+2.6}$ & $17.5_{-3.9}^{+4.6}$ & $-0.43_{-0.24}^{+0.18}$ & $1.47_{-0.07}^{+0.63}$ \\
\hline HSCJ2216-0016 (PN) & $<4.7$ & $<3.9$ & $<5.5$ & - & - \\
\hline " (MOS1) & $<2.3$ & $<2.3$ & $<2.3$ & - & - \\
\hline " (MOS2 & $<2.3$ & $<2.3$ & $<2.3$ & - & - \\
\hline
\end{tabular}

Notes. Net counts and associated uncertainties are computed by deriving probability distribution function of net counts with the method of Weisskopf et al. (2007).

number of sources per bin. For visual purposes only, we did not include upper limits (i.e., QSOs not detected in the X-rays) from Lusso \& Risaliti (2016), which would populate preferentially the steep $\alpha_{\text {ox }}$ regime.

In order to check if the $\alpha_{\mathrm{ox}}$ values we found are in agreement with those expected from literature relations, we first note that the probability that a source is observed with an $\alpha_{\text {ox }}$ flatter or steeper than the expectation from a reference relation (we assumed the Just et al. 2007 one, based on optically selected QSOs as is our sample) due to random fluctuations only can be described by a binomial distribution, with probability of "success" $p=0.5$ (i.e., we expect half of the sample to be above the relation), and number of trials $n=25$ (i.e., the sample size). Assuming the two extreme cases in which upper limits on $\alpha_{\text {ox }}$ are treated as a detection (i.e., $x=13$ sources above the relation) or represent sources intrinsically below the relation 
F. Vito et al.: The X-ray properties of $z>6$ QSOs

Table 4. X-ray fluxes, luminosities, and derived properties for our Chandra and archival sample of $z>6$ QSOs.

\begin{tabular}{|c|c|c|c|c|c|c|}
\hline \multirow[t]{2}{*}{ ID } & \multicolumn{3}{|c|}{$\begin{array}{c}F \\
{\left[10^{-15} \mathrm{erg} \mathrm{cm}^{-2} \mathrm{~s}^{-1}\right]}\end{array}$} & \multirow[t]{2}{*}{$\begin{array}{c}L_{2-10 \mathrm{keV}} \\
{\left[10^{44} \mathrm{erg} \mathrm{s}^{-1}\right]}\end{array}$} & \multirow[t]{2}{*}{$\alpha_{\mathrm{ox}}$} & \multirow[t]{2}{*}{$\Delta \alpha_{\mathrm{ox}}$} \\
\hline & SB & HB & FB & & & \\
\hline \multicolumn{7}{|c|}{ New observations } \\
\hline CFHQS J0050+3445 & $1.07_{-0.43}^{+0.59}$ & $1.48_{-0.80}^{+1.19}$ & $2.46_{-0.83}^{+1.05}$ & $6.68_{-2.70}^{+3.67}$ & $-1.71_{-0.09}^{+0.07}$ & $-0.02_{-0.09}^{+0.07}$ \\
\hline VIK J0109-3047 & $<0.47$ & $<0.63$ & $<0.56$ & $<3.29$ & $<-1.67$ & $<-0.04$ \\
\hline PSO J036+03 & $1.26_{-0.55}^{+0.77}$ & $<3.53$ & $2.49_{-0.95}^{+1.27}$ & $8.20_{-3.57}^{+5.05}$ & $-1.77_{-0.10}^{+0.08}$ & $-0.05_{-0.10}^{+0.08}$ \\
\hline VIK J0305-3150 & $<0.59$ & $<1.73$ & $<1.31$ & $<3.79$ & $<-1.72$ & $<-0.06$ \\
\hline SDSS J0842+1218 & $0.75_{-0.38}^{+0.56}$ & $<2.26$ & $1.30_{-0.66}^{+0.94}$ & $4.34_{-2.17}^{+3.26}$ & $-1.81_{-0.12}^{+0.09}$ & $-0.11_{-0.12}^{+0.09}$ \\
\hline PSO J167-13 & $<0.32$ & $<2.44$ & $<1.39$ & $<2.21$ & $<-1.72$ & $<-0.09$ \\
\hline CFHQS J1509-1749 & $1.67_{-0.62}^{+0.82}$ & $1.81_{-1.00}^{+1.47}$ & $2.28_{-0.91}^{+1.21}$ & $10.34_{-3.86}^{+5.10}$ & $-1.71_{-0.08}^{+0.07}$ & $0.01_{-0.08}^{+0.07}$ \\
\hline CFHQS J1641+3755 & $6.43_{-0.98}^{+1.07}$ & $2.85_{-0.93}^{+1.17}$ & $10.65_{-1.49}^{+1.63}$ & $33.39_{-5.07}^{+5.56}$ & $-1.28_{-0.03}^{+0.03}$ & $0.35_{-0.03}^{+0.03}$ \\
\hline PSO J338+29 & $0.78_{-0.29}^{+0.39}$ & $<1.61$ & $1.43_{-0.52}^{+0.66}$ & $5.92_{-2.22}^{+2.96}$ & $-1.64_{-0.08}^{+0.07}$ & $0.01_{-0.08}^{+0.07}$ \\
\hline SDSS J2310+1855 & $1.22_{-0.59}^{+0.88}$ & $<3.85$ & $2.29_{-1.05}^{+1.49}$ & $6.93_{-3.34}^{+5.02}$ & $-1.87_{-0.11}^{+0.09}$ & $-0.12_{-0.11}^{+0.09}$ \\
\hline \multicolumn{7}{|c|}{ Archival observations } \\
\hline SDSS J0100+2802 & $7.28_{-0.47}^{+0.50}$ & $4.10_{-0.95}^{+1.03}$ & $14.09_{-0.91}^{+0.94}$ & $47.64_{-3.08}^{+3.27}$ & $-1.76_{-0.01}^{+0.01}$ & $0.07_{-0.01}^{+0.01}$ \\
\hline ATLAS J0142-3327 & $2.26_{-0.68}^{+0.82}$ & $<2.92$ & $3.75_{-1.14}^{+1.40}$ & $13.69_{-4.11}^{+4.98}$ & $-1.76_{-0.06}^{+0.05}$ & $-0.01_{-0.06}^{+0.05}$ \\
\hline CFHQS J0210-0456 & $<4.83$ & $<5.162$ & $<6.62$ & $<29.31$ & $<-1.13$ & $<0.44$ \\
\hline CFHQS J0216-0455 & $<0.52$ & $<0.92$ & $<0.70$ & $<2.70$ & $<-1.21$ & $<0.24$ \\
\hline SDSS J0303-0019 & $<15.9$ & $<108.5$ & $<46.00$ & $<97.70$ & $<-1.09$ & $<0.54$ \\
\hline SDSS J1030+0524 & $1.82_{-0.20}^{+0.21}$ & $2.05_{-0.30}^{+0.33}$ & $3.84_{-0.34}^{+0.36}$ & $10.77_{-1.18}^{+1.27}$ & $-1.68_{-0.02}^{+0.02}$ & $0.03_{-0.02}^{+0.02}$ \\
\hline SDSS J1048+4637 & $0.77_{-0.37}^{+0.56}$ & $<2.66$ & $1.23_{-0.61}^{+0.97}$ & $4.25_{-2.05}^{+3.08}$ & $-1.86_{-0.11}^{+0.09}$ & $-0.15_{-0.11}^{+0.09}$ \\
\hline ULAS J1120+0641 & $0.73_{-0.15}^{+0.16}$ & $0.59_{-0.33}^{+0.39}$ & $1.41_{-0.30}^{+0.32}$ & $6.07_{-1.25}^{+1.33}$ & $-1.72_{-0.04}^{+0.03}$ & $-0.03_{-0.04}^{+0.03}$ \\
\hline SDSS J1148+5251 & $2.17_{-0.40}^{+0.49}$ & $2.39_{-0.70}^{+0.87}$ & $4.51_{-0.70}^{+0.80}$ & $12.94_{-2.39}^{+2.69}$ & $-1.74_{-0.03}^{+0.03}$ & $-0.00_{-0.03}^{+0.03}$ \\
\hline SDSS J1306+0356 & $3.25_{-0.31}^{+0.33}$ & $3.93_{-0.71}^{+0.81}$ & $6.93_{-0.60}^{+0.62}$ & $17.06_{-1.63}^{+1.73}$ & $-1.57_{-0.02}^{+0.02}$ & $0.12_{-0.02}^{+0.02}$ \\
\hline ULAS J1342+0928 & $1.70_{-0.50}^{+0.61}$ & $1.82_{-0.80}^{-0.111}$ & $3.52_{-0.89}^{+0.00}$ & $14.96_{-4.40}^{-5.03}$ & $\begin{array}{r}-0.02 \\
-1.57_{-0.06}^{+0.05}\end{array}$ & $0.12_{-0.06}^{-0.02}$ \\
\hline SDSS J1602+4228 & $7.09_{-1.39}^{+1.58}$ & $4.87_{-2.24}^{+3.16}$ & $13.05_{-2.50}^{+2.89}$ & $37.04_{-7.28}^{+8.40}$ & $-1.46_{-0.04}^{+0.00}$ & $0.24_{-0.04}^{+0.03}$ \\
\hline SDSS J1623+3112 & $0.90_{-0.39}^{+0.55}$ & $2.92_{-1.41}^{+2.12}$ & $2.60_{-0.88}^{+1.50}$ & $5.08_{-2.22}^{+3.13}$ & $-1.73_{-0.10}^{+0.04}$ & $-0.05_{-0.10}^{+0.08}$ \\
\hline SDSS J1630+4012 & $1.92_{-0.50}^{+0.61}$ & $3.06_{-1.21}^{+1.66}$ & $4.35_{-0.97}^{+1.14}$ & $9.92_{-2.58}^{+3.12}$ & $-1.57_{-0.05}^{+0.05}$ & $0.09_{-0.05}^{+0.05}$ \\
\hline HSC J2216-0016 & $<1.01$ & $<4.04$ & $<2.43$ & $<5.92$ & $<-1.25$ & $<0.27$ \\
\hline
\end{tabular}

Notes. Errors account for the uncertainties on the net counts only.

(i.e., $x=9$ ), a binomial test returns probabilities of the observed or more extreme configurations given the expected configuration of $P=0.50$ and $P=0.11$, respectively. If we do not consider the four sources with weak upper limits on $\alpha_{\text {ox }}$, which do not provide useful information, we find $n=21$ and $x=9$, corresponding to $P=0.33$. According to these values, we do not find evidence supporting a significant variation of $\alpha_{\mathrm{ox}}\left(L_{2500 \AA}\right)$ with redshift from this basic assessment.

This result can also be assessed by computing the difference between the observed $\alpha_{\mathrm{ox}}$ and the value expected from the UV luminosity, according to the relation of Just et al. (2007), that is, $\Delta \alpha_{\mathrm{ox}}=\alpha_{\mathrm{ox}}$ (observed) $-\alpha_{\mathrm{ox}}\left(L_{2500 \AA}\right)$ as a function of redshift (Fig. 4). If $\alpha_{\text {ox }}\left(L_{2500 \AA}\right)$ does not vary significantly with redshift, we expect the $\Delta \alpha_{\text {ox }}$ distribution of our sample to be consistent with the distribution of the sample used by Just et al. (2007) to fit their relation. We test this null hypothesis (i.e., that the two $\Delta \alpha_{\mathrm{ox}}$ distributions are drawn from the same population) using the univariate methods (Feigelson \& Nelson 1985) included in ASURV Rev. 1.2 (Isobe \& Feigelson 1990; Lavalley et al. 1992), which allows accounting for censored data (i.e., sources undetected in X-rays). The null-hypothesis probabilities for the several tests we ran are reported in Table 5. According to these tests, the $\Delta \alpha_{\text {ox }}$ distribution of our sample is consistent with those of lower-redshift samples collected from the literature. Finally, we computed the Kaplan-Meier estimator for the distribution function of the $\Delta \alpha_{\mathrm{ox}}$ parameters of the considered samples. Results are summarized in Table $6^{10}$. Following Steffen et al. (2006, see their Sect. 3.5), we can estimate roughly the allowed fractional variation of the typical UV-to-X-ray flux ratio in QSOs as $\delta r / r=2.606 \ln (10) \alpha_{\text {ox }} \approx 6 \delta \alpha_{\text {ox }}=0.16$ at $1 \sigma$, where $r=f_{v}(2500 \AA) / f_{v}(2 \mathrm{keV})$ and $\delta \alpha_{\mathrm{ox}}$ is the allowed variation of $\alpha_{\text {ox }}$, which we approximated with the uncertainty on the mean of $\Delta \alpha_{\text {ox }}$ computed with the Kaplan-Meier estimator. This estimate may be somewhat optimistic, as, for instance, we did

${ }^{10}$ As reported in the ASURV manual, the Kaplan-Meier estimator requires the censoring to be random. Formally, this is not the case for our sample, as the censored variable, $\Delta \alpha_{\mathrm{ox}}$, is directly related to the QSO luminosities, and less-luminous QSOs are more likely not to be detected. However, in addition to the luminosity of the QSOs, the censoring of $\Delta \alpha_{\mathrm{ox}}$ is due to the flux limit of the observations (i.e., the exposure times) and the distances of the QSOs, which thus help to randomize the censoring distribution. 


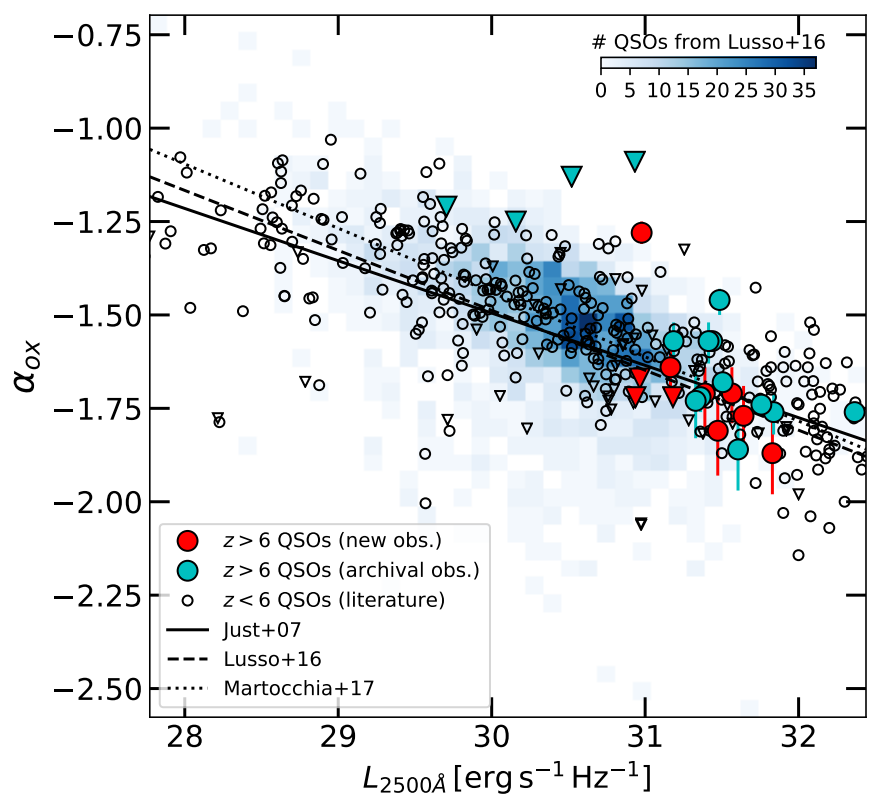

Fig. 3. $\alpha_{\text {ox }}$ vs. $L_{2500 \AA}$ for $z \geq 6$ QSOs. We compared our results with a compilation of optically selected QSOs at lower redshifts (Shemmer et al. 2006a; Steffen et al. 2006; Just et al. 2007; Lusso \& Risaliti 2016). Downward pointing triangles represent upper limits. We also show the best-fitting relations of Just et al. (2007), Lusso \& Risaliti (2016), and Martocchia et al. (2017). For visual purposes, we do not plot X-ray undetected sources included in the Lusso \& Risaliti (2016) sample.

not take into account the uncertainties on the Just et al. (2007)

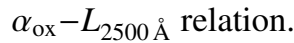

In Fig. 5 we also compare the distribution of $\Delta \alpha_{\text {ox }}$ of our $z>6$ QSOs with the sample of $z \approx 2$ QSOs presented in Gibson et al. (2008, improved sample B; see Footnote 3 of Ni et al. 2018), which has been carefully selected to discard BALQSOs, and includes only X-ray detected QSOs. The two distributions are broadly consistent, again pointing toward a lack of a significant evolution of $\alpha_{\text {ox }}$ with redshift. We do not find a significant deviation of $\Delta \alpha_{\mathrm{ox}}$ also limiting the tests to QSOs at the highest redshifts $(z>6.5)$ in our sample, although we note that the size of such a subsample is too small (7 QSOs, 3 of which undetected) to derive strong conclusions.

Based on the apparently non-evolving QSO $L_{\mathrm{UV}}-L_{\mathrm{X}}$ relation across cosmic time, Risaliti \& Lusso (2019) propose the use of QSOs up to $z \approx 5$ as standard candles to infer cosmological parameters, finding evidence for a deviation from the concordance $\Lambda C D M$ model. In this respect, since type Ia supernovae are detected up to $z \approx 1.4$ only, QSOs are particularly useful in the distant universe.

We do not find evidence supporting a significant correlation between $\Delta \alpha_{\mathrm{ox}}$ and $M_{\mathrm{BH}}$, bolometric luminosity, or $\lambda_{\mathrm{Edd}}$ : Spearman's test returned $\rho=-0.10$ and $P=0.66, \rho=0.04$ and $P=0.84$, and $\rho=0.24$ and $P=0.29$, respectively. We underline that $\Delta \alpha_{\mathrm{ox}}$ factors out the dependence of $\alpha_{\mathrm{ox}}$ with UV luminosity, which also enters into the computation of $M_{\mathrm{BH}}$ and bolometric luminosity, and it is thus a better parameter to use when checking for any potential correlation with such quantities.

QSO emission variability is potentially a significant source of uncertainty affecting the derived values of $\alpha_{\mathrm{ox}}$ and $\Delta \alpha_{\mathrm{ox}}$ (e.g., Gibson \& Brandt 2012; Vagnetti et al. 2013). For instance, Shemmer et al. (2005) detected X-ray flux variability of a factor of $\approx 4$ for SDSS J02310-728 at $z=5.41$ over a rest-frame period of $\approx 73$ days. Nanni et al. (2018) found evidence for strong variability affecting the emission of SDSS J1030+0524 at $z=6.308$ (see also Shemmer et al. 2005): its X-ray flux increased by a factor of $\approx 2.5$ from an XMM-Newton observation in 2003 to the 2017 Chandra dataset analyzed in this work, corresponding to a variation of $\Delta \alpha_{\mathrm{ox}}$ of \pm 0.16 . As also discussed in Sect. 3.3, we do not find other similar cases among the few other QSOs covered by multiple observations.

\subsection{Bolometric corrections}

Figure 6 presents the X-ray luminosities of $z>6$ QSOs plotted against their bolometric luminosities. We compare these with the sample of lower-luminosity Type 1 AGN selected in the XMM-COSMOS survey of Lusso et al. (2010), and with QSO samples with luminosities similar to or larger than those of our high-redshift sample (Feruglio et al. 2014; Banerji et al. 2015; Cano-Díaz et al. 2012; Martocchia et al. 2017; Ricci et al. 2017; Vito et al. 2018). In particular, our sample populates a luminosity regime in this plane poorly sampled before. The positions of our $z>6$ sources confirm the trend of increasing bolometric correction $K_{\mathrm{bol}}=L_{\mathrm{bol}} / L_{\mathrm{X}}$ with bolometric luminosity, from $K_{\text {bol }} \approx 10-100$ at $\log L_{\text {bol }} \lesssim 46.5$ to $K_{\text {bol }} \approx 100-1000$ at $\log \frac{L_{\mathrm{bol}}}{\mathrm{erg} \mathrm{s}^{-1}} \gtrsim 46.5$, in agreement with previous works. We note that the bolometric luminosities of our type 1 QSOs are derived from the UV luminosities as described in Sect. 2.3, with typical relative uncertainties of $\sim 7 \%$. Thus, the bolometric corrections found are byproducts of the relation shown in Fig. 3.

\subsection{Spectral analysis}

\subsubsection{Individual sources}

We performed a basic spectral analysis for individual sources in our sample, considering only those detected in at least one energy band, in order to compare the resulting parameters with those derived from hardness-ratio and aperture photometry analyses (Tables 3 and 4). Spectra, response matrices, and ancillary files were extracted as described in Sect. 3.1. We fit the spectra with XSPEC v12.9.0n (Arnaud 1996) ${ }^{11}$. We used the $W$-statistic ${ }^{12}$, which extends the Cash (1979) statistic in the case of background-subtracted data. In the case of a source observed by more than one instrument, we performed a joint spectral analysis using all the available spectra. Due to the generally limited photon counting statistics, we assumed a simple power-law model, and included Galactic absorption along the line of sight of each source (Kalberla et al. 2005). The photon index and the power-law normalization are the only free parameters. Notwithstanding the simplicity of the model, the fit does not converge for SDSS J0842+1218 (which has $\approx 3$ net counts), which is thus not considered hereafter. For two other sources, PSO J036+03, SDSS1048+5251, and SDSS J2310+1855, the fit converges but returns only an upper limit on the power-law normalization, and thus on flux and luminosity.

Best-fit parameters are reported in Table 7. Although the uncertainties are typically large, the results derived from spectral and hardness-ratio analyses are consistent, suggesting that the procedures used in the previous sections are robust. The luminosity derived for PSO J338+29 from spectral analysis is significantly larger than the value found from photometric analysis

\footnotetext{
11 https://heasarc.gsfc.nasa.gov/xanadu/xspec/

12 https://heasarc.gsfc.nasa.gov/xanadu/xspec/manual/ XSappendixStatistics.html
} 


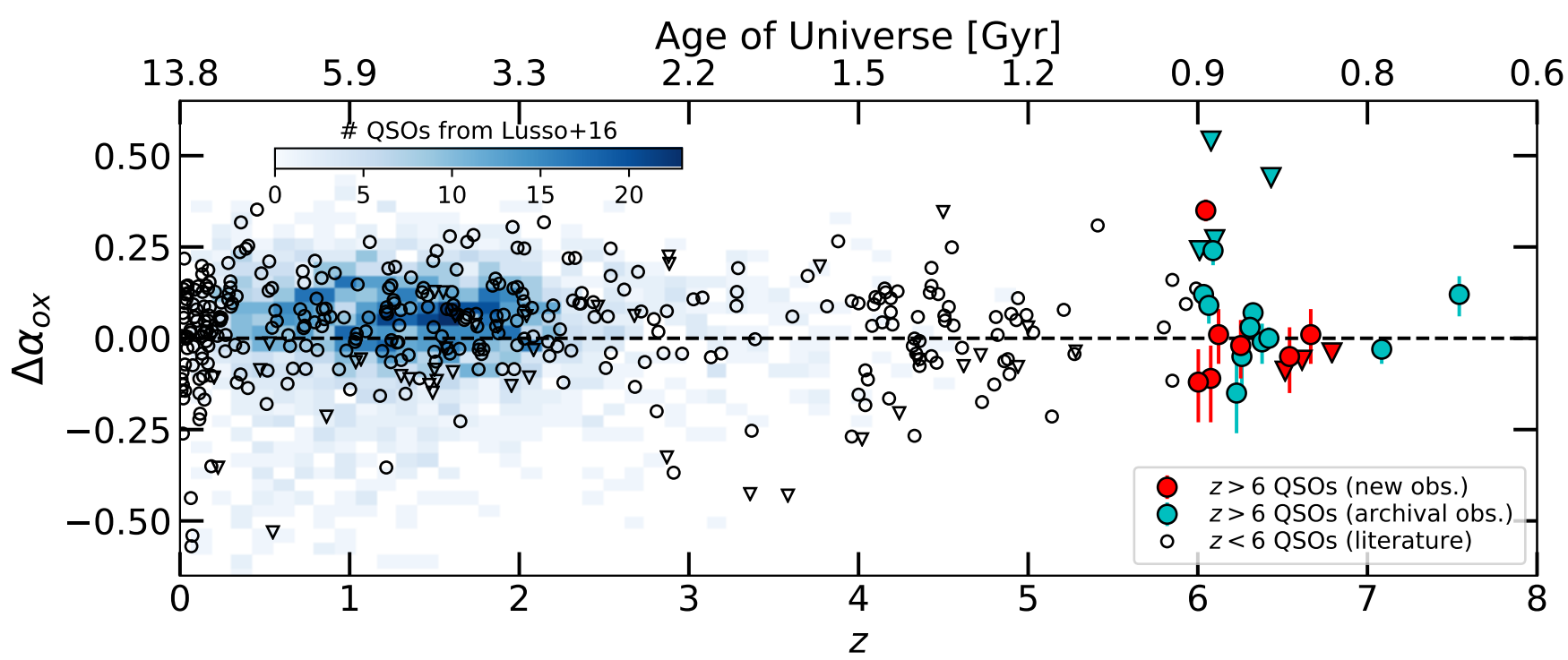

Fig. 4. $\Delta \alpha_{\text {ox }}$ vs. redshift for $z \geq 6$ QSOs. We compared our results with a compilation of QSOs at lower redshifts (see Fig. 3). Downward-pointing triangles represent upper limits. The horizontal dashed line corresponds to $\Delta \alpha_{\mathrm{ox}}=0$.

Table 5. Probabilities that $\Delta \alpha_{\text {ox }}$ distributions (including censored values) of our sample and samples taken from literature (Just et al. 2007 and Lusso \& Risaliti 2016) are drawn from same parent population.

\begin{tabular}{lc}
\hline \hline Test & $P$ \\
\hline \multicolumn{1}{c}{ Just et al. (2007) sample $(z<6)$} \\
\hline Gehan's generalized Wilcoxon test \\
(permutation variance) & 0.31 \\
(hypergeometric variance) & 0.30 \\
Logrank test & 0.67 \\
Peto and Peto generalized Wilcoxon test & 0.32 \\
Peto and Prentice generalized Wilcoxon test & 0.30 \\
\hline \multicolumn{2}{c}{ Lusso \& Risaliti (2016) sample $(z<6)$} \\
\hline Gehan's generalized Wilcoxon test \\
(permutation variance) \\
(hypergeometric variance) \\
Logrank test \\
Peto and Peto generalized Wilcoxon test \\
Peto and Prentice generalized Wilcoxon test & 0.49 \\
\hline
\end{tabular}

Table 6. Results of Kaplan-Meier estimator for distribution function of $\Delta \alpha_{\mathrm{ox}}$ for our $z>6$ sample and lower-redshift samples from Just et al. (2007) and Lusso \& Risaliti (2016).

\begin{tabular}{lccc}
\hline \hline Mean & \multicolumn{3}{c}{ Percentiles } \\
\cline { 2 - 4 }$\Delta \alpha_{\text {ox }}$ & $25 \%$ & $50 \%$ & $75 \%$ \\
\hline \multicolumn{4}{c}{$z>6$ sample (this work) } \\
$0.005 \pm 0.026$ & -0.113 & -0.016 & 0.056 \\
\hline \multicolumn{4}{c}{ Just et al. $(2007)$ sample $(z<6)$} \\
$0.005 \pm 0.009$ & -0.066 & 0.033 & 0.107 \\
\hline \multicolumn{4}{c}{ Lusso \& Risaliti (2016) sample $(z<6)$} \\
$-0.036 \pm 0.011$ & -0.084 & 0.021 & 0.101 \\
\hline
\end{tabular}

(Table 4), where we assumed $\Gamma=2.0$. This is due to the extremely steep best-fitting photon index derived from spectral analysis, likely due to the limited photon-counting statistics.

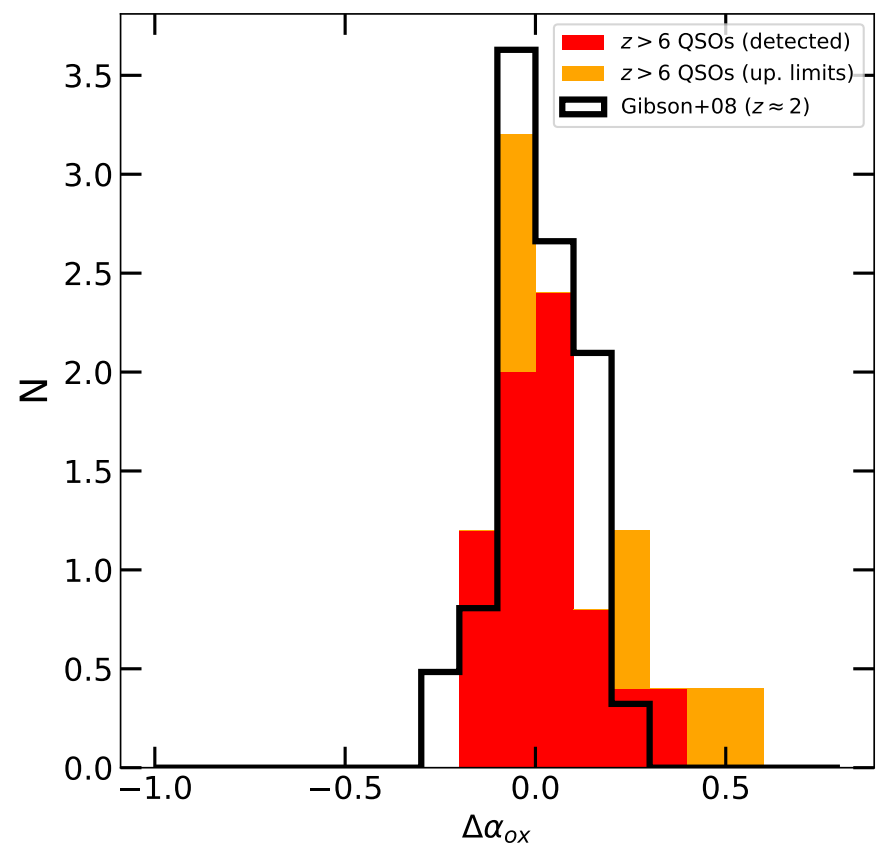

Fig. 5. Normalized histogram of $\Delta \alpha_{\text {ox }}$ for our sample of $z>6$ QSOs (detections and upper limits are represented with red and orange histograms, respectively). We compared it with the sample of $z \approx 2$ QSOs presented as sample B of Gibson et al. (2008).

\subsubsection{Joint spectral analysis}

We performed a joint spectral analysis to estimate the average photon index of sources detected in at least one energy band (18 sources). We removed the 6 QSOs with a total of more than 30 net counts in their spectra, for which results from individual spectral fitting are reported in Sect. 4.3.1, as they would dominate the spectral-fit results. We used a single power-law model with photon index free to vary, but linked among the datasets, to fit jointly the remaining 12 sources $(\approx 115$ net counts in the $0.5-7 \mathrm{keV}$ band) and added Galactic absorption appropriate to each source. We found a best-fitting, average photon 


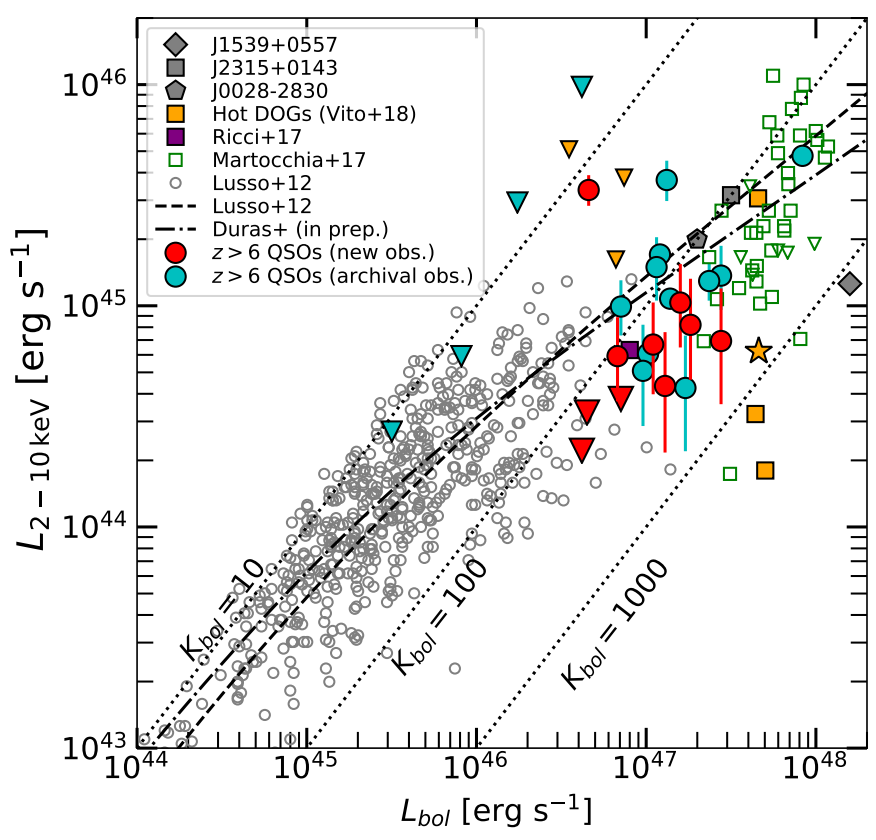

Fig. 6. X-ray vs. bolometric luminosity of our sample of $z>6$ QOSs (red and cyan symbols). We compared our results with the compilation of lower luminosity QSOs of Lusso et al. (2012, empty gray circles). We also add the sample of luminous QSOs from Martocchia et al. (2017, green symbols), the Hot DOG samples of Vito et al. (2018, orange symbols; the orange star represents the stacked result of several Hot DOGs undetected in the X-rays) and Ricci et al. (2017, purple square), and results for some individual hyperluminous QSOs (filled gray symbols; see Martocchia et al. 2017 for their luminosities Feruglio et al. 2014; Banerji et al. 2015; Cano-Díaz et al. 2012). The dashed and dash-dotted black curves are the best-fitting relation of Lusso et al. (2012) and Duras et al. (in prep.), respectively. Downward pointing triangles represent upper limits. Diagonal dotted lines mark the loci of constant bolometric correction.

index $\Gamma=2.20_{-0.34}^{+0.39}$ (errors at the $90 \%$ c.l. corresponding to $\Delta W=2.7 ; \Gamma=2.20_{-0.20}^{+0.22}$ with errors at the $68 \%$ c.l. corresponding to $\Delta W=1.0)^{13}$. Repeating the joint spectral analysis for the 6 QSOs with $>30$ net counts $(\approx 746$ net counts in total $)$, we found an average $\Gamma=2.13_{-0.13}^{+0.13}( \pm 0.08$ at the $68 \%$ c.1.). Considering only QSOs at $z>6.5$, ULAS1120+0641 is detected with $>30$ counts, and its best-fitting photon index is $\Gamma \approx 2$ (see Table 7). Joint spectral analysis of the other three $z>6.5$ QSOs detected in the X-rays $\left(\approx 23\right.$ net counts in total) returns $\Gamma=2.66_{-0.78}^{+0.92}$ $\left(\Gamma=2.66_{-0.50}^{+0.54}\right.$ with errors at the $68 \%$ c.l. $)$.

All of these values are slightly steeper than, although still consistent with, that found by Nanni et al. (2017) for $z>5.7$ QSOs, thus including a subsample of our sources (i.e., $\Gamma=$ $1.93_{-0.29}^{+0.30}$, and with the findings of Piconcelli et al. (2005), Vignali et al. (2005), Shemmer et al. (2006a), and Just et al. (2007) at lower redshifts (Fig. 7). Thus, we conclude there is no strong evidence supporting a significant systematic variation of $\Gamma$ in our sample, although there are hints of a steepening of the typical QSO photon index at $z>6$.

The observed-frame $0.5-7 \mathrm{keV}$ band corresponds to restframe energies at $z>6$ where a possible Compton-reflection component would peak in the X-ray spectra of QSOs. We did not account for this component in the spectral fitting, due to

\footnotetext{
13 The average derived through joint spectral analysis is by construction weighted by the number of counts of each spectrum, and thus depends on a complex combination of source fluxes and exposure times.
}

Table 7. Best-fitting parameters derived from spectral analysis of individual sources (see Sect. 4.3.1).

\begin{tabular}{|c|c|c|c|}
\hline ID & $\Gamma$ & $\begin{array}{c}F_{0.5-2 \mathrm{keV}} \\
{\left[10^{-15} \mathrm{erg} \mathrm{cm}^{-2} \mathrm{~s}^{-1}\right]}\end{array}$ & $\begin{array}{c}L_{2-10 \mathrm{keV}} \\
{\left[10^{44} \mathrm{erg} \mathrm{s}^{-1}\right]}\end{array}$ \\
\hline \multicolumn{4}{|c|}{ New observations } \\
\hline CFHQS J0050+3445 & $2.12_{-1.17}^{+2.01}$ & $1.45_{-0.89}^{+1.57}$ & $8.20_{-5.03}^{+8.88}$ \\
\hline PSO J036+03 & $2.10_{-1.50}^{+2.23}$ & $<3.05$ & $<20.53$ \\
\hline CFHQS J1509-1749 & $1.73_{-1.10}^{+1.29}$ & $1.42_{-0.91}^{+1.72}$ & $7.69_{-4.93}^{+9.31}$ \\
\hline CFHQS J1641+3755 ${ }^{(*)}$ & $2.36_{-0.47}^{+0.50}$ & $6.36_{-1.81}^{+2.26}$ & $39.20_{-11.21}^{+13.90}$ \\
\hline PSO J338+29 & $4.52_{-2.12}^{+2.57}$ & $1.41_{-0.83}^{+1.30}$ & $57.31_{-33.64}^{+53.18}$ \\
\hline SDSS J2310+1855 & $3.18_{-3.67}^{+2.65}$ & $<3.34$ & $<35.85$ \\
\hline \multicolumn{4}{|c|}{ Archival observations } \\
\hline SDSS J0100+2802 $2^{(*)}$ & $2.52_{-0.22}^{+0.23}$ & $7.71_{-1.02}^{+1.10}$ & $67.55_{-8.93}^{+9.63}$ \\
\hline ATLAS J0142-3327 & $2.03_{-1.10}^{+1.28}$ & $1.98_{-1.03}^{+1.43}$ & $12.14_{-6.37}^{+8.52}$ \\
\hline SDSS J1030+0524 ${ }^{(*)}$ & $1.83_{-0.28}^{+0.29}$ & $1.76_{-0.38}^{+0.44}$ & $9.55_{-2.04}^{+2.41}$ \\
\hline SDSS1048+5251 & $1.84_{-1.56}^{+1.88}$ & $<1.52$ & $<7.79$ \\
\hline ULAS J1120+0641 ${ }^{(*)}$ & $2.08_{-0.64}^{+0.74}$ & $0.68_{-0.28}^{+0.48}$ & $6.56_{-3.27}^{+3.59}$ \\
\hline SDSS J1148+5251 ${ }^{(*)}$ & $1.65_{-0.48}^{+0.50}$ & $1.96_{-0.64}^{+0.83}$ & $9.78_{-3.21}^{+4.11}$ \\
\hline SDSS J1306+0356 ${ }^{(*)}$ & $1.83_{-0.25}^{+0.26}$ & $3.22_{-0.49}^{+5.44}$ & $15.60_{-2.38}^{+2.64}$ \\
\hline ULAS J1342+0928 & $1.97_{-0.92}^{+1.16}$ & $1.73_{-0.88}^{+1.33}$ & $14.95_{-7.60}^{+11.51}$ \\
\hline SDSS J1602+4228 & $2.19_{-0.61}^{+0.74}$ & $6.89_{-2.10}^{+2.62}$ & $39.43_{-12.03}^{+14.99}$ \\
\hline SDSS J1623+3112 & $0.91_{-1.03}^{+2.40}$ & $0.89_{-0.59}^{+1.07}$ & $3.00_{-1.99}^{+3.62}$ \\
\hline SDSS J1630+4012 & $1.90_{-0.69}^{+0.92}$ & $2.04_{-7.87}^{+1.05}$ & $10.03_{-3.87}^{+5.15}$ \\
\hline
\end{tabular}

Notes. Errors correspond to the $90 \%$ confidence level for one parameter of interest (Avni 1976). ${ }^{(*)}$ These sources have $>30$ net counts in the $0.5-7 \mathrm{keV}$ band.

the small number of total counts preventing the use of relatively complex models. However, we note that the reflection component in the X-ray spectra of luminous Type-1 QSOs has been found to be generally weak both in the local universe (e.g., Comastri et al. 1992; Piconcelli et al. 2005) and at high redshift ( $z>4$, e.g., Shemmer et al. 2005). Moreover, a strong reflection component would tend to flatten systematically the observed effective photon index, in contrast with our results.

Performing joint spectral analysis on subsamples of QSOs divided on the basis of their Eddington ratios, we do not find any significant trend of $\Gamma$ with $\lambda_{\text {Edd }}$. However, this may be due to the small sample size, and the large uncertainties affecting the single-epoch black hole masses and the best-fitting photon indices.

In order to place a basic upper limit on the average column density, we added an XSPEC zwabs component and repeated the joint fit of QSOs with $>30$ net counts. We left both the photon index and the column density free to vary, but linked them among the spectra, and fixed the redshift to the appropriate value for each QSO. The best-fitting parameters are $\Gamma=2.17_{-0.14}^{+0.22}$ and $N_{\mathrm{H}}<9 \times 10^{22} \mathrm{~cm}^{-2}$ at the $90 \%$ confidence level (see Fig. 8 for the confidence contours). The upper limit on $N_{\mathrm{H}}$ is dominated by the high-redshift nature of the sources, which causes the photoelectric cutoff to shift below Chandra observed energy bands even for possible moderately high values of column density.

\subsection{Comments on individual QSOs}

\subsubsection{PSO J167-13}

This QSO $(z=6.515)$ falls slightly below our detection threshold in the hard band $\left(P_{B}=0.989\right)$. We then checked whether we could detect it by restricting the detection energy range to the $2-5 \mathrm{keV}$ band. This choice is motivated by the drop of the 


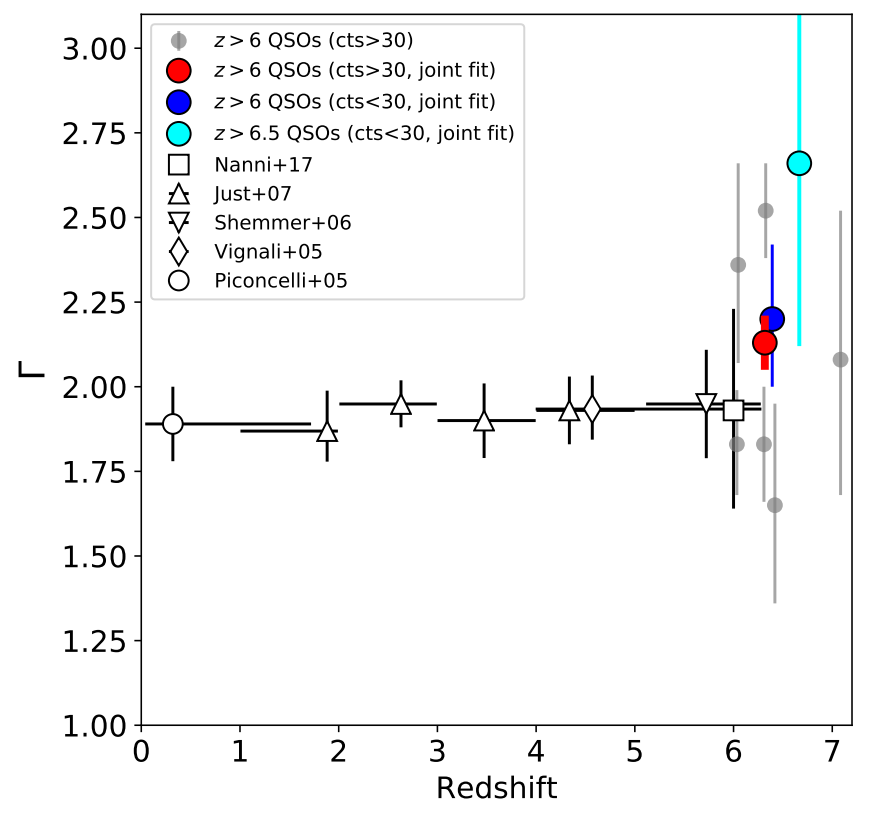

Fig. 7. Photon index as function of redshift. We report the individual best-fitting values for sources with $>30$ total net counts (gray symbols), the results derived from joint spectral analysis of QSOs with $>30,<30$ net counts, and of $z>6.5$ QSOs (red, blue, and cyan circles, respectively, plotted at the median redshift of each subsample), and the average photon indices derived by Piconcelli et al. (2005), Vignali et al. (2005), Shemmer et al. (2006b), Just et al. (2007), and Nanni et al. (2017) for optically selected luminous QSOs at different redshifts. Errors are at the $68 \%$ confidence level.

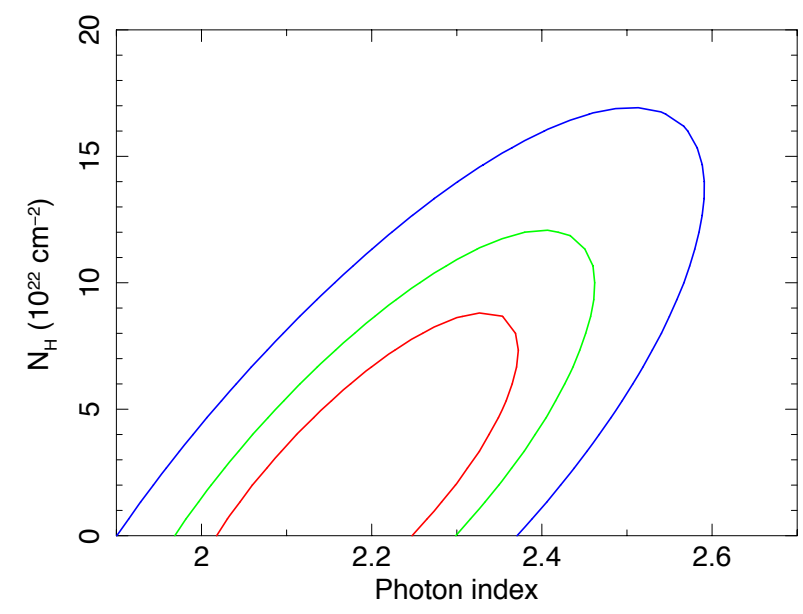

Fig. 8. Confidence contours at $68 \%, 90 \%$, and $99 \%$ confidence levels (red, green, and blue curves, respectively) of best-fitting column density and photon index derived from joint spectral analysis of QSOs with >30 counts (see Sect. 4.3.2).

Chandra effective area and the relatively high background level at higher energies. Moreover, observed energies $E>5 \mathrm{keV}$ correspond to $E>37.5 \mathrm{keV}$ in the QSO rest frame, where the number of emitted X-ray photons is limited due to the QSO power-law spectrum.

An X-ray source is significantly detected $\left(P_{B}=4 \times 10^{-4}\right)$ with $2.9_{-1.4}^{+2.1}$ net counts in the $2-5 \mathrm{keV}$ band in an $R=1 \mathrm{arcsec}$ circular region (Fig. 9). The centroid of the X-ray emission shows an offset of $\approx 1$ arcsec with respect to the optical position of the QSO, but with a positional uncertainty of $1.2 \mathrm{arcsec}$ at the $90 \%$ confidence level. Considering the lack of counts detected

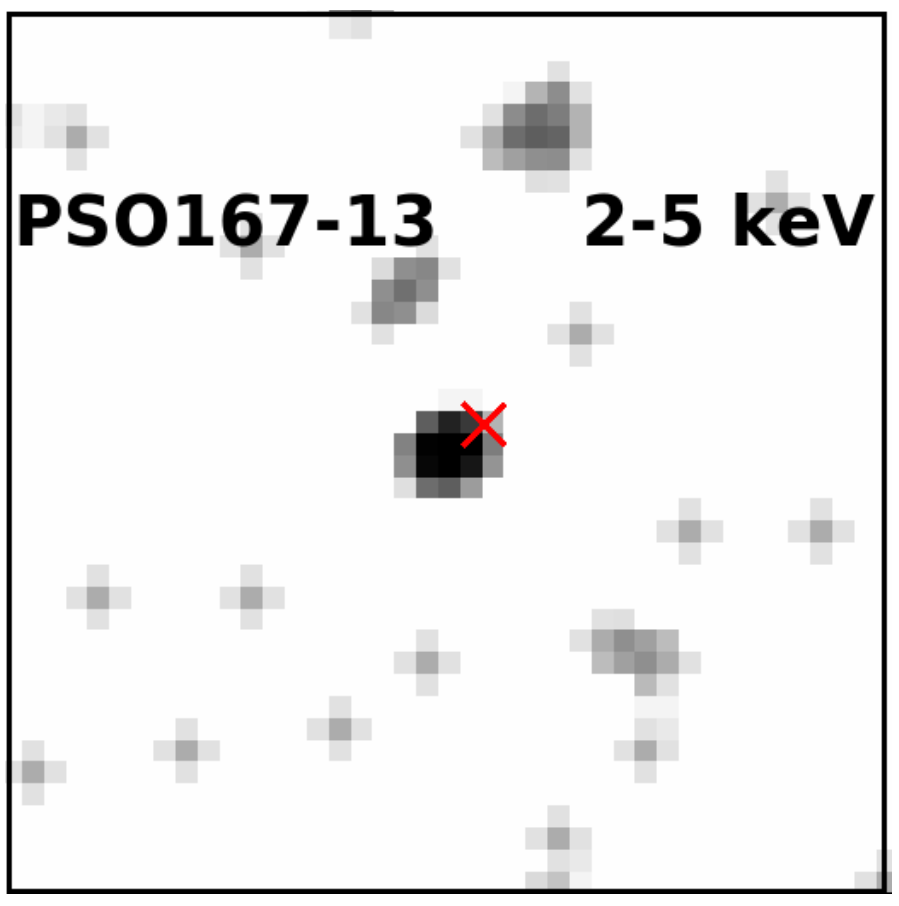

Fig. 9. Smoothed $2-5 \mathrm{keV}$ image $\left(40 \times 40\right.$ pixels; $\left.\approx 20^{\prime \prime} \times 20^{\prime \prime}\right)$ of PSO167-13. The red cross marks the optical position of the QSO.

in the soft band, following the procedure used in Sect. 3.3, we derived HR $>0.47$ and $\mathrm{HR}>0.08$ at the $68 \%$ and $90 \%$ confidence levels, corresponding to $\Gamma_{\text {eff }}<0.55$ and $\Gamma_{\text {eff }}<1.54$, respectively. This very hard spectrum at $z=6.515$, assuming an intrinsic $\Gamma=2$ spectrum, corresponds to lower limits on the obscuring column density of $N_{\mathrm{H}}>2 \times 10^{24} \mathrm{~cm}^{-2}$ and $N_{\mathrm{H}}>6 \times 10^{23} \mathrm{~cm}^{-2}$ at the $68 \%$ and $90 \%$ confidence levels, respectively. Therefore this object is the first heavily obscured QSO candidate at $z>6$, with the intriguing property of being an optically classified Type 1 QSO.

An ALMA sub-mm observation (Willott et al. 2017) revealed the presence of a close galaxy companion from the rest-frame UV and [C II] position of the QSO $(0.9 \mathrm{arcsec}$; i.e., $\approx 5 \mathrm{kpc}$ in projection at the redshift of the QSO), and by $\Delta v \approx-270 \mathrm{~km} \mathrm{~s}^{-1}$ (i.e., $\Delta z \approx 0.007$ ) in velocity space. The offset between the X-ray centroid and the [C II] position of this galaxy is only $\approx 0.15$ arcsec. A thorough investigation and discussion of this system has been presented separately (Vito et al. 2019).

\subsubsection{VIK0305-3150}

Similarly to PSO167-13, VIK0305-3150 $(z=6.047)$ is slightly below our detection threshold both in the hard and full bands. We thus repeated the analysis restricting the energy bands to $2-5 \mathrm{keV}$ and $0.5-5 \mathrm{keV}$. We nominally detected this QSO in a $R=1 \mathrm{arcsec}$ circular region in the $2-5 \mathrm{keV}$ band with $P_{B}=6.6 \times 10^{-3}$, but with a very limited number of net counts $\left(1.9_{-1.1}^{+1.8}\right)$. The detection in the $0.5-5 \mathrm{keV}$ band is more solid $\left(P_{B}=1.1 \times 10^{-3}\right)$ with $2.8_{-1.4}^{+2.1}$ net counts (Fig. 10). Repeating the same hardness-ratio analysis as done above for PSO167-13, we found $\mathrm{HR}>0.00$ and $\mathrm{HR}>-0.39$, corresponding to $\Gamma_{\text {eff }}<0.96$ and $\Gamma_{\text {eff }}<1.93$ at the $68 \%$ and $90 \%$ confidence levels, respectively. Assuming an intrinsic $\Gamma=1.9$, the nominal obscuring column density is $N_{\mathrm{H}}>1 \times 10^{24} \mathrm{~cm}^{-2}$ at the $68 \%$ confidence level, but it is not constrained at the $90 \%$ confidence level. 


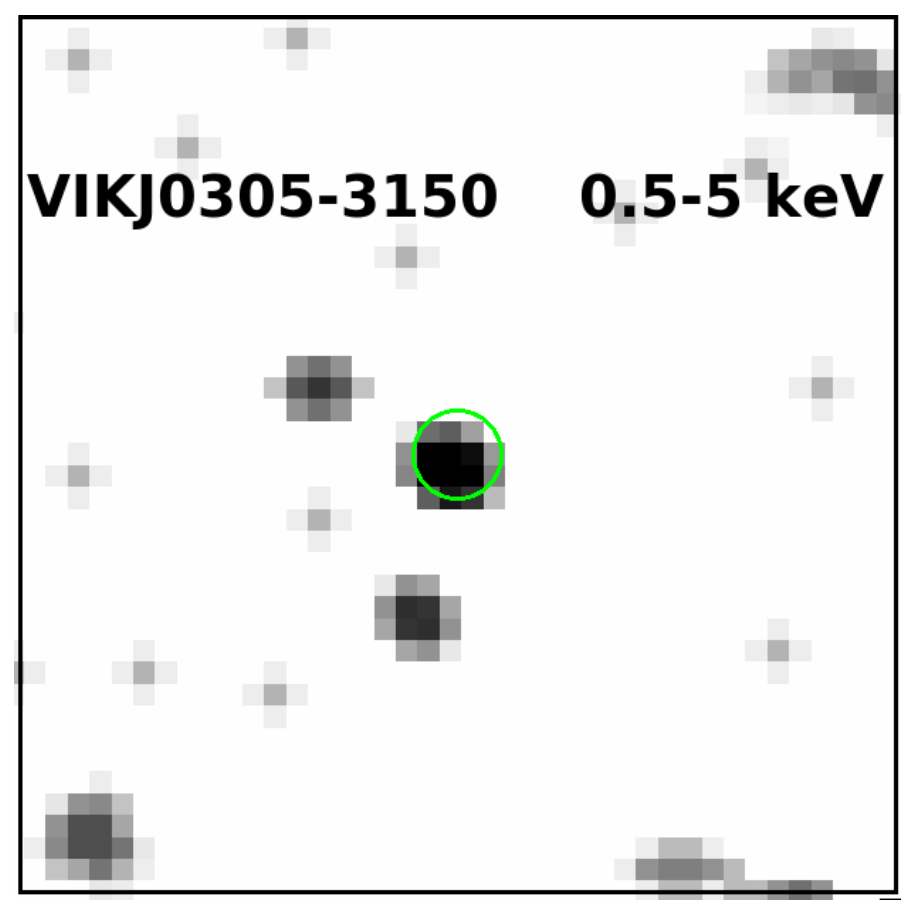

Fig. 10. Smoothed $0.5-5 \mathrm{keV}$ image $\left(40 \times 40\right.$ pixels; $\left.\approx 20^{\prime \prime} \times 20^{\prime \prime}\right)$ of VIK J0305-3150. The green circle has a radius $R=1 \mathrm{arcsec}$.

\subsubsection{CFHQS J1641+3755}

This radio-quiet $(R<10.5) \mathrm{QSO}$ at $z=6.047$ has one of the lowest bolometric luminosities $\left(\log \frac{L_{\text {bol }}}{L_{0}}=13.1\right)$ and smallest black hole masses $\left(\log \frac{M_{\mathrm{BH}}}{M_{\mathrm{O}}}=8.4\right)$ among the $z>6$ QSO sample, resulting in a high Eddington ratio $\left(\lambda_{\mathrm{Edd}}=1.5\right)$. While the bolometric correction is usually found to anti-correlate with the Eddington ratio both observationally (e.g., Lusso et al. 2012) and theoretically (e.g., Meier 2012; Jiang et al. 2019, but see also Castelló-Mor et al. 2017), CFHQS J1641+3755 is the second most X-ray luminous $z>6$ QSO, resulting in a bolometric correction $K_{\text {bol }} \approx 13$. This is also reflected in a quite flat $\alpha_{\mathrm{ox}}=-1.28$. Considering an rms $=0.2$ for the $\alpha_{\mathrm{ox}}-L_{\mathrm{UV}}$ relation in the $L_{\mathrm{UV}}$ luminosity range where CFHQS J1641+3755 lies (Steffen et al. 2006), this QSO is a $\approx 1.8 \sigma$ outlier.

Its $\approx 50$ net counts allowed us to constrain with a reasonable accuracy its photon index, which, as expected considering its high Eddington ratio (e.g., Brightman et al. 2013; Fanali et al. 2013), is quite steep $\left(\Gamma=2.36_{-0.47}^{+0.50}\right)$. Consistent results for the estimated photon index and X-ray luminosity are found from the photometric and spectral analyses (see Tables 3 , 4, and 7).

\subsubsection{WLQs and BALQSOs}

As discussed at the end of Sect. 2.3, among our sample are BALQSO and WLQ candidates, which are often associated with weak X-ray emission. The UV spectral quality of several of these objects prevents us from securely including them in one of these classes, but we note that the two QSOs with the most negative $\Delta \alpha_{\text {ox }}$ values (Table 4) are a WLQ candidate (J2310+1855) and a known BALQSO (J1048+4637). The remaining candidates have $\Delta \alpha_{\text {ox }}$ values consistent with the rest of the sample. Detected WLQ and BALQSO candidates do not show particularly flat photon indices (see Table 7), which might suggest the presence of a significant level of X-ray absorption.

\section{Conclusions}

We have presented new Chandra observations of $10 z>6$ QSOs, selected to be radio quiet and to have virial black-hole mass estimates from Mg II line measurements. With this sample, we more than triple the number of QSOs at $z>6.5$ with existing sensitive $\mathrm{X}$-ray coverage. In particular, five of the targets have UV magnitudes $-26.2<M_{1450 \AA}<-25.6$, and are thus the least luminous $z>6$ QSOs targeted with sensitive Xray observations. We detected $7 / 10$ of our new targets in at least one standard energy band, and 2 additional QSOs discarding $E>5 \mathrm{keV}$. Adding archival observations at $z>6$, we could study the X-ray properties of a statistically significant sample of 25 QSO in the first Gyr of the universe. Our main results are the following:

- From photometric analysis, we constrained or derived upper limits on the X-ray luminosity of $z>6$ QSOs, and their basic spectral shape, modeled with a simple power law. Consistent results are found from spectral analysis of individual bright sources, although the derived individual best-fitting photon indices have large uncertainties. See Sects. 3.3 and 4.3.

- We do not find evidence for a significant evolution of the relation between QSO UV and X-ray luminosity, as traced by the $\alpha_{\text {ox }}$ parameter. The luminosities of $z>6$ QSOs are consistent with relations found at lower redshift for optically selected QSOs (e.g., Just et al. 2007; Lusso \& Risaliti 2016), implying that the coronal emission becomes less important compared with disk emission at high luminosity also at $z>6$. See Sect. 4.1.

- We do not find significant correlations between $\alpha_{\text {ox }}$ and black-hole mass or Eddington ratio, once the dependence of all of these quantities with the QSO UV luminosity is taken into account. See Sect. 4.1.

- We confirm the trend of increasing bolometric correction with increasing luminosity from $K_{\text {bol }} \approx 10-100$ at $\log \frac{L_{\text {bol }}}{L_{L_{\odot}}} \lesssim$ 46.5 to $K_{\text {bol }} \approx 100-1000$ at $\log \frac{L_{\text {bol }}}{L_{L_{\odot}}} \gtrsim 46.5$, for the first time at $z>6$. In particular, our sample populates the luminosity region between moderate luminosity QSOs and ultraluminous QSOs, currently poorly sampled. See Sect. 4.2.

- We perform a basic spectral analysis of sources with $>30$ net counts, and derived typical photon indices $\Gamma \approx 1.6-2.5$. Joint spectral analysis of fainter sources returned an average value $\left(\Gamma=2.13_{-0.13}^{+0.13}\right.$ and $\Gamma=2.20_{-0.34}^{+0.39}$, for sources with $>30$ and $<30$ net counts, respectively) slightly steeper, but still consistent with, typical photon indices of lower redshift QSOs. This result again supports a scenario in which the accretiondisk/hot-corona structure does not evolve strongly from low redshift to $z>6$. See Sect. 4.3.

- Two of the three undetected targets could be detected by restricting the energy range to avoid background-dominated regions $(E>5 \mathrm{keV})$. In particular, one of these, PSO167-13, presents a very hard spectrum, consistent with a large obscuring column density, and it is thus the first heavily obscured QSO candidate at $z>6$. See Sect. 4.4.

Only $\approx 25$ of the $z>6$ QSOs have been currently observed in the $\mathrm{X}$-rays, while the number of known high-redshift QSOs is continuously growing. Moreover, over the coming $\approx 10-20$ years, wide-field surveys (e.g., Euclid, eROSITA, LSST, SUMIREHSC, and WFIRST) are expected to push the QSO redshift frontier far into the reionization era, detecting hundreds of accreting SMBHs at $z \approx 7-10$ (e.g., Brandt \& Vito 2017). Studying QSO properties in the first few $10^{8}$ years of the Universe will be extremely important to understand some of the major open issues in modern astrophysics, such as the formation 
and early growth of SMBHs, their interplay with proto-galaxies, the formation of the first structures, and the mechanisms responsible for the reionization of the Universe. Observing larger samples of high-redshift QSOs with Chandra and XMM-Newton will provide key X-ray information on their small-scale accretion physics, even in the presence of heavy obscuration, and will pave the way for future X-ray observatories, such as Athena, Lynx, and AXIS. It is especially important to assess if the hints we find for steepening X-ray power-law spectra, and high associated Eddington ratios, become stronger at still higher redshifts. Targeting of $z>8$ QSOs in the next decades will take advantage of the tightest constraints we have placed on the $\mathrm{X}$-ray properties of the $z \approx 6-7$ QSO population. In particular, realistic exposuretime estimates can be computed on the basis of the lack of a strong evolution of the $L_{X}-L_{\mathrm{UV}}$ relation up to the highest redshifts which can be probed currently.

Acknowledgements. We thank the anonymous referee for useful feedback. We thank L. Jiang for providing the near-IR spectrum of SDSSJ2310+1855, A Moretti for his help in reducing Swift data, C. Willott for useful discussions, E. Picconcelli and S. Martocchia for providing bolometric luminosities of the WISSH QSOs, and F. Duras for providing their functional form of the bolometric correction curve. FV acknowledges financial support from CONICYT and CASSACA through the Fourth call for tenders of the CAS-CONICYT Fund, CONICYT grants Basal-CATA AFB-170002 (FV, FEB), the Ministry of Economy, Development, and Tourism's Millennium Science Initiative through gran IC120009, awarded to The Millennium Institute of Astrophysics, MAS (FEB). WNB acknowledges support from CXC grant G08-19076X and NASA ADP grant 80NSSC18K0878. BL acknowledges financial support from the National Key R\&D Program of China grant 2016YFA0400702 and National Natural Science Foundation of China grant 11673010 . We acknowledge financial contribution from the agreement ASI-INAF n.2017-14-H.O.

\section{References}

Arnaud, K. A. 1996, in Astronomical Data Analysis Software and Systems V, eds. G. H. Jacoby, \& J. Barnes, ASP Conf. Ser., 101, 17

Avni, Y. 1976, ApJ, 210, 642

Bañados, E., Decarli, R., Walter, F., et al. 2015a, ApJ, 805, L8

Bañados, E., Venemans, B. P., Morganson, E., et al. 2015b, ApJ, 804, 118

Bañados, E., Venemans, B. P., Decarli, R., et al. 2016, ApJS, 227, 11

Bañados, E., Venemans, B. P., Mazzucchelli, C., et al. 2018a, Nature, 553, 473

Bañados, E., Connor, T., Stern, D., et al. 2018b, ApJ, 856, L25

Banerji, M., Alaghband-Zadeh, S., Hewett, P. C., \& McMahon, R. G. 2015 , MNRAS, 447, 3368

Becker, R. H., White, R. L., \& Helfand, D. J. 1995, ApJ, 450, 559

Beloborodov, A. M. 2017, ApJ, 850, 141

Brandt, W. N., \& Alexander, D. M. 2015, A\&ARv, 23, 1

Brandt, W. N., \& Vito, F. 2017, Astron. Nachr., 338, 241

Brandt, W. N., Schneider, D. P., Fan, X., et al. 2002, ApJ, 569, L5

Brightman, M., Silverman, J. D., Mainieri, V., et al. 2013, MNRAS, 433, 2485

Broos, P. S., Feigelson, E. D., Townsley, L. K., et al. 2007, ApJS, 169, 353

Cano-Díaz, M., Maiolino, R., Marconi, A., et al. 2012, A\&A, 537, L8

Carnall, A. C., Shanks, T., Chehade, B., et al. 2015, MNRAS, 451, L16

Cash, W. 1979, ApJ, 228, 939

Castelló-Mor, N., Kaspi, S., Netzer, H., et al. 2017, MNRAS, 467, 1209

Chehade, B., Carnall, A. C., Shanks, T., et al. 2018, MNRAS, 478, 1649

Comastri, A., Setti, G., Zamorani, G., et al. 1992, ApJ, 384, 62

Condon, J. J., Cotton, W. D., Greisen, E. W., et al. 1998, AJ, 115, 1693

Decarli, R., Walter, F., Venemans, B. P., et al. 2018, ApJ, 854, 97

De Rosa, G., Decarli, R., Walter, F., et al. 2011, ApJ, 739, 56

De Rosa, G., Venemans, B. P., Decarli, R., et al. 2014, ApJ, 790, 145

Diamond-Stanic, A. M., Fan, X., Brandt, W. N., et al. 2009, ApJ, 699, 782

Fan, X., Strauss, M. A., Gunn, J. E., et al. 1999, ApJ, 526, L57

Fan, X., Narayanan, V. K., Lupton, R. H., et al. 2001, AJ, 122, 2833

Fan, X., Strauss, M. A., Schneider, D. P., et al. 2003, AJ, 125, 1649

Fan, X., Hennawi, J. F., Richards, G. T., et al. 2004, AJ, 128, 515

Fan, X., Wang, F., Yang, J., et al. 2019, ApJ, 870, L11

Fanali, R., Caccianiga, A., Severgnini, P., et al. 2013, MNRAS, 433, 648

Farrah, D., Priddey, R., Wilman, R., Haehnelt, M., \& McMahon, R. 2004, ApJ, 611, L13

Feigelson, E. D., \& Nelson, P. I. 1985, ApJ, 293, 192

Feruglio, C., Bongiorno, A., Fiore, F., et al. 2014, A\&A, 565, A91
Galeev, A. A., Rosner, R., \& Vaiana, G. S. 1979, ApJ, 229, 318

Gallagher, S. C., Brandt, W. N., Chartas, G., et al. 2006, ApJ, 644, 709

Gallerani, S., Zappacosta, L., Orofino, M. C., et al. 2017, MNRAS, 467, 3590

Gibson, R. R., \& Brandt, W. N. 2012, ApJ, 746, 54

Gibson, R. R., Brandt, W. N., \& Schneider, D. P. 2008, ApJ, 685, 773

Gibson, R. R., Jiang, L., Brandt, W. N., et al. 2009, ApJ, 692, 758

Haardt, F., \& Maraschi, L. 1991, ApJ, 380, L51

Isobe, T., \& Feigelson, E. D. 1990, Bull. Am. Astron. Soc., 22, 917

Iwamuro, F., Kimura, M., Eto, S., et al. 2004, ApJ, 614, 69

Jiang, L., Fan, X., Annis, J., et al. 2008, AJ, 135, 1057

Jiang, L., McGreer, I. D., Fan, X., et al. 2016, ApJ, 833, 222

Jiang, Y.-F., Stone, J., \& Davis, S. W. 2019, ApJ, 880, 67

Jin, C., Ward, M., \& Done, C. 2012, MNRAS, 422, 3268

Just, D. W., Brandt, W. N., Shemmer, O., et al. 2007, ApJ, 665, 1004

Kalberla, P. M. W., Burton, W. B., Hartmann, D., et al. 2005, A\&A, 440, 775

Kellermann, K. I., Sramek, R., Schmidt, M., Shaffer, D. B., \& Green, R. 1989, AJ, 98, 1195

Kurk, J. D., Walter, F., Fan, X., et al. 2007, ApJ, 669, 32

Kurk, J. D., Walter, F., Fan, X., et al. 2009, ApJ, 702, 833

Lavalley, M. P., Isobe, T., \& Feigelson, E. D. 1992, Bull. Am. Astron. Soc., 24, 839

Luo, B., Brandt, W. N., Alexander, D. M., et al. 2014, ApJ, 794, 70

Luo, B., Brandt, W. N., Hall, P. B., et al. 2015, ApJ, 805, 122

Lusso, E., \& Risaliti, G. 2016, ApJ, 819, 154

Lusso, E., \& Risaliti, G. 2017, A\&A, 602, A79

Lusso, E., Comastri, A., Vignali, C., et al. 2010, A\&A, 512, A34

Lusso, E., Comastri, A., Simmons, B. D., et al. 2012, MNRAS, 425, 623

Marchese, E., Della Ceca, R., Caccianiga, A., et al. 2012, A\&A, 539, A48

Marlar, A., Shemmer, O., Anderson, S. F., et al. 2018, ApJ, 865, 92

Martocchia, S., Piconcelli, E., Zappacosta, L., et al. 2017, A\&A, 608, A51

Matsuoka, Y., Onoue, M., Kashikawa, N., et al. 2016, ApJ, 828, 26

Matsuoka, Y., Onoue, M., Kashikawa, N., et al. 2018a, PASJ, 70, S35

Matsuoka, Y., Iwasawa, K., Onoue, M., et al. 2018b, ApJS, 237, 5

Matsuoka, Y., Strauss, M. A., Kashikawa, N., et al. 2018c, ApJ, 869, 150

Matsuoka, Y., Onoue, M., Kashikawa, N., et al. 2019, ApJ, 872, L2

Mazzucchelli, C., Bañados, E., Venemans, B. P., et al. 2017, ApJ, 849, 91

Meier, D. L. 2012, Black Hole Astrophysics: The Engine Paradigm (Berlin: Springer)

Meyer, R. A., Bosman, S. E. I., \& Ellis, R. S. 2019, MNRAS, 487, 3305

Miller, B. P., Brandt, W. N., Schneider, D. P., et al. 2011, ApJ, 726, 20

Moretti, A., Campana, S., Mineo, T., et al. 2005, in UV, X-Ray, and Gamma-Ray Space Instrumentation for Astronomy XIV, ed. O. H. W. Siegmund, SPIE Conf. Ser., 5898, 360

Mortlock, D. J., Warren, S. J., Venemans, B. P., et al. 2011, Nature, 474, 616

Nanni, R., Vignali, C., Gilli, R., Moretti, A., \& Brandt, W. N. 2017, A\&A, 603, A128

Nanni, R., Gilli, R., Vignali, C., et al. 2018, A\&A, 614, A121

Ni, Q., Brandt, W. N., Luo, B., et al. 2018, MNRAS, 480, 5184

Omont, A., Willott, C. J., Beelen, A., et al. 2013, A\&A, 552, A43

Pâris, I., Petitjean, P., Aubourg, É., et al. 2018, A\&A, 613, A51

Park, T., Kashyap, V. L., Siemiginowska, A., et al. 2006, ApJ, 652, 610

Piconcelli, E., Jimenez-Bailón, E., Guainazzi, M., et al. 2005, A\&A, 432, 15

Planck Collaboration XIII. 2016, A\&A, 594, A13

Plotkin, R. M., Shemmer, O., Trakhtenbrot, B., et al. 2015, ApJ, 805, 123

Pons, E., McMahon, R. G., Simcoe, R. A., et al. 2019, MNRAS, 484, 5142

Reed, S. L., McMahon, R. G., Martini, P., et al. 2017, MNRAS, 468, 4702

Reed, S. L., Banerji, M., Becker, G. D., et al. 2019, MNRAS, 487, 1874

Ricci, C., Assef, R. J., Stern, D., et al. 2017, ApJ, 835, 105

Risaliti, G., \& Lusso, E. 2019, Nat. Astron., 195

Risaliti, G., Young, M., \& Elvis, M. 2009, ApJ, 700, L6

Salvestrini, F., Risaliti, G., Bisogni, S., et al. 2019, A\&A, in press, https://doi.org/10.1051/0004-6361/201935491

Selsing, J., Fynbo, J. P. U., Christensen, L., \& Krogager, J. K. 2016, A\&A, 585, A87

Shemmer, O., Brandt, W. N., Vignali, C., et al. 2005, ApJ, 630, 729

Shemmer, O., Brandt, W. N., Schneider, D. P., et al. 2006a, ApJ, 644, 86

Shemmer, O., Brandt, W. N., Netzer, H., Maiolino, R., \& Kaspi, S. 2006b, ApJ, 646, L29

Shemmer, O., Brandt, W. N., Netzer, H., Maiolino, R., \& Kaspi, S. 2008, ApJ, 682,81

Shemmer, O., Brandt, W. N., Paolillo, M., et al. 2017, ApJ, 848, 46

Shen, Y. 2013, Bull. Astron. Soc. India, 41, 61

Shen, Y., \& Kelly, B. C. 2012, ApJ, 746, 169

Shen, Y., Brandt, W. N., Richards, G. T., et al. 2016, ApJ, 831, 7

Shen, Y., Wu, J., Jiang, L., et al. 2019, ApJ, 873, 35

Simpson, C., Martínez-Sansigre, A., Rawlings, S., et al. 2006, MNRAS, 372, 741

Steffen, A. T., Strateva, I., Brandt, W. N., et al. 2006, AJ, 131, 2826 
Tang, J.-J., Goto, T., Ohyama, Y., et al. 2017, MNRAS, 466, 4568 Trakhtenbrot, B., Ricci, C., Koss, M. J., et al. 2017a, MNRAS, 470, 800 Trakhtenbrot, B., Volonteri, M., \& Natarajan, P. 2017b, ApJ, 836, L1 Vagnetti, F., Antonucci, M., \& Trevese, D. 2013, A\&A, 550, A71 Vanden Berk, D. E., Richards, G. T., Bauer, A., et al. 2001, AJ, 122, 549 Venemans, B. P., McMahon, R. G., Walter, F., et al. 2012, ApJ, 751, L25 Venemans, B. P., Findlay, J. R., Sutherland, W. J., et al. 2013, ApJ, 779, 24 Venemans, B. P., Bañados, E., Decarli, R., et al. 2015, ApJ, 801, L11 Venemans, B. P., Walter, F., Zschaechner, L., et al. 2016, ApJ, 816, 37 Venemans, B. P., Walter, F., Decarli, R., et al. 2017, ApJ, 851, L8 Vestergaard, M., \& Osmer, P. S. 2009, ApJ, 699, 800 Vignali, C., Brandt, W. N., \& Schneider, D. P. 2003, AJ, 125, 433 Vignali, C., Brandt, W. N., Schneider, D. P., \& Kaspi, S. 2005, AJ, 129, 2519 Vito, F., Brandt, W. N., Stern, D., et al. 2018, MNRAS, 474, 4528 Vito, F., Brandt, W. N., Bauer, F. E., et al. 2019, A\&A, 628, L6 Wang, R., Carilli, C. L., Neri, R., et al. 2010, ApJ, 714, 699
Wang, R., Wagg, J., Carilli, C. L., et al. 2011, ApJ, 739, L34 Wang, R., Wagg, J., Carilli, C. L., et al. 2013, ApJ, 773, 44 Wang, R., Wu, X.-B., Neri, R., et al. 2016, ApJ, 830, 53 Wang, F., Fan, X., Yang, J., et al. 2017, ApJ, 839, 27

Wang, F., Yang, J., Fan, X., et al. 2018a, ApJ, submitted [arXiv:1810.11926] Wang, F., Yang, J., Fan, X., et al. 2018b, ApJ, 869, L9 Weisskopf, M. C., Wu, K., Trimble, V., et al. 2007, ApJ, 657, 1026 Willott, C. J., Delorme, P., Omont, A., et al. 2007, AJ, 134, 2435 Willott, C. J., Delorme, P., Reylé, C., et al. 2009, AJ, 137, 3541 Willott, C. J., Albert, L., Arzoumanian, D., et al. 2010, AJ, 140, 546 Willott, C. J., Omont, A., \& Bergeron, J. 2013, ApJ, 770, 13 Willott, C. J., Bergeron, J., \& Omont, A. 2017, ApJ, 850, 108 Woods, T. E., Agarwal, B., Bromm, V., et al. 2019, PASA, 36, e027 Wu, J., Brandt, W. N., Comins, M. L., et al. 2010, ApJ, 724, 762 Wu, X.-B., Wang, F., Fan, X., et al. 2015, Nature, 518, 512 Yang, J., Wang, F., Fan, X., et al. 2019, AJ, 157, 236 